\title{
Effect of Three Water Regimes on the Physiological and Anatomical Structure of Stem and Leaves of Different Citrus Rootstocks with Distinct Degrees of Tolerance to Drought Stress
}

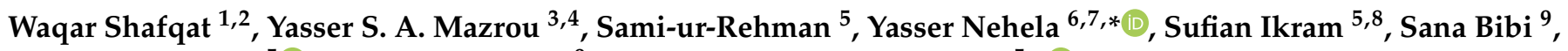 \\ Summar A. Naqvi ${ }^{5}\left(\mathbb{D}\right.$, Mansoor Hameed ${ }^{9}$ and Muhammad Jafar Jaskani ${ }^{5, *} * \mathbb{C}$ \\ 1 Indian River Research and Education Center, Horticultural Sciences Department, University of Florida, \\ 2199 South Rock Road, Fort Pierce, FL 34945, USA; waqar.shafqat@ufl.edu \\ 2 Department of Forestry, Mississippi State University, Starkville, MS 39762, USA \\ 3 Business Administration Department, Community College, King Khalid University, Guraiger, \\ Abha 62529, Saudi Arabia; ymazrou@kku.edu.sa or yasser.mazroua@agr.tanta.edu.eg \\ 4 Department of Agriculture Economic, Faculty of Agriculture, Tanta University, Tanta 31527, Egypt \\ 5 Institute of Horticultural Science, University of Agriculture, Faisalabad 38000, Pakistan; \\ samiurrehman96@gmail.com (S.-u.-R.); chsufian1@yahoo.com (S.I.); summar.naqvi@uaf.edu.pk (S.A.N.) \\ 6 Department of Agricultural Botany, Faculty of Agriculture, Tanta University, Tanta 31527, Egypt \\ 7 Citrus Research and Education Center, Department of Plant Pathology, University of Florida, 700 Experiment \\ Station Road, Lake Alfred, FL 33850, USA \\ check for \\ updates \\ Citation: Shafqat, W.; Mazrou, Y.S.A.; \\ Sami-ur-Rehman; Nehela, Y.; Ikram, \\ S.; Bibi, S.; Naqvi, S.A.; Hameed, M.; \\ 8 Institute of Vegetables and Flowers, Chinese Academy of Agricultural Sciences (CAAS), \\ No. 12 Zhongguancun South Street, Haidian District, Beijing 100081, China \\ 9 Department of Botany, University of Agriculture, Faisalabad 38040, Pakistan; sanisana446@gmail.com (S.B.); \\ hameedmansoor@yahoo.com (M.H.) \\ * Correspondence: yasser.nehela@ufl.edu (Y.N.); jjaskani@uaf.edu.pk (M.J.J.)
} Jaskani, M.J. Effect of Three Water Regimes on the Physiological and Anatomical Structure of Stem and Leaves of Different Citrus Rootstocks with Distinct Degrees of Tolerance to Drought Stress. Horticulturae 2021, 7, 554. https://doi.org/10.3390/ horticulturae7120554

Academic Editor: Yanyou Wu

Received: 21 October 2021

Accepted: 1 December 2021

Published: 6 December 2021

Publisher's Note: MDPI stays neutral with regard to jurisdictional claims in published maps and institutional affiliations.

Copyright: (c) 2021 by the authors. Licensee MDPI, Basel, Switzerland. This article is an open access article distributed under the terms and conditions of the Creative Commons Attribution (CC BY) license (https:/ / creativecommons.org/licenses/by/ $4.0 /)$.

\begin{abstract}
Citrus is grown globally throughout the subtropics and semi-arid to humid tropics. Abiotic stresses such as soil water deficit negatively affect plant growth, physiology, biochemistry, and anatomy. Herein, we investigated the effect(s) of three water regimes (control, moderate drought, and severe drought) on the physiological and anatomical structure of 10 different citrus rootstocks with different degrees of tolerance to drought stress. Brazilian sour orange and Gadha dahi performed well by avoiding desiccation and maintaining plant growth, plant water status, and biochemical characters, while Rangpur Poona nucellar (C. limonia) and Sunki $\times$ bentake were the most sensitive rootstocks at all stress conditions. At severe water stress, the highest root length $(24.33 \pm 0.58)$, shoot length $(17.00 \pm 1.00)$, root moisture content $(57.67 \pm 1.53)$, shoot moisture content $(64.59 \pm 1.71)$, and plant water potential $(-1.57 \pm 0.03)$ was observed in tolerant genotype, Brazilian sour orange. Likewise, chlorophyll $a(2.70 \pm 0.06)$, chlorophyll $b(0.87 \pm 0.06)$ and carotenoids $(0.69 \pm 0.08)$ were higher in the same genotype. The lowest $\mathrm{H}_{2} \mathrm{O}_{2}$ content $(77.00 \pm 1.00)$ and highest proline content $(0.51 \pm 0.06)$ were also recorded by Brazilian sour orange. The tolerance mechanism of tolerant genotypes was elucidated by modification in anatomical structures. Stem anatomy at severe drought, $27.5 \%$ increase in epidermal cell thickness, $25.4 \%$ in vascular bundle length, $30.5 \%$ in xylem thickness, $27.7 \%$ in the phloem cell area, $8 \%$ in the pith cell area, and $43.4 \%$ in cortical thickness were also observed in tolerant genotypes. Likewise, leaf anatomy showed an increase of $27.9 \%$ in epidermal cell thickness, $11.4 \%$ in vascular bundle length, $21 \%$ in xylem thickness, and $15 \%$ in phloem cell area in tolerant genotypes compared with sensitive ones. These modifications in tolerant genotypes enabled them to maintain steady nutrient transport while reducing the risk of embolisms, increasing water-flow resistance, and constant transport of nutrients across.
\end{abstract}

Keywords: drought stress; citrus; oxidative stress; proline; photosynthesis; water potential; vascular bundle modifications 


\section{Introduction}

Citrus fruit crops in the family Rutaceae have the largest fruit industry globally [1,2]. Rootstock choice and selection for the citrus scion variety are the most valuable decisions for growers for the implication of better yield and quality with other valuable characters. Citrus rootstock controls the physiological, biochemical, morphological, and genetic characteristics of scion cultivars grafted on selected rootstocks through the rootstock scion interaction pathway [3]. Fruit juice quality and tree productivity of scion cultivars are also affected by rootstock characters. Rootstock has a significant impact on nutrition, horticultural and pathological traits of citrus cultivars, growth, vigor, stress resistance, and fruit quality of the scion [4-7]. Rootstock controls translocation of water and nutrients from the soil and distributes among different up ground plant organs, which were also disturbed negatively by the impact of stresses $[8,9]$.

Citrus plants affected by several biotic (fungal, viral, and bacterial diseases) and abiotic (water deficit, heat, flooding, and salinity) stresses exhibit wide-ranging losses in citrus production $[10,11]$. Cultivars of genus Citrus are grown in a climate of wide range due to evergreens and perennial tree crops [12]. The capacity to tolerate the unfavorable climatic conditions of the citrus crop is high but the estimated yield loss due to environmental abiotic stresses in citrus is up to $82 \%$ [13]. Climate change conditioning causes a global rise in temperature and limited the availability of freshwater for the crop. Meanwhile, crop consumption is increasing rapidly resulting in reduced levels of groundwater ultimately limiting productivity [14]. Global warming due to climate change caused frequent extreme water deficit conditions, an important component for global agriculture [15,16]. Abiotic stresses are interlinked with each other in which soil water deficit/drought is a serious environmental factor, which frequently limits the growth and productivity of important crop species [17]. Supply in water restriction can severely limit plant cell division, plant growth, fruit development, and fruit production [18,19]. Soil water deficit for long-term events causes permanent negative changes in the plant which can be in response to previous stress. Water deficit conditions can be developed at different phenological stages which can change physiological and molecular processes of the plant [20].

Plant responses to water stress are mediated by changes in root growth pattern and stomatal closure at moderate stress resulting in reduced $\mathrm{CO}_{2}$ intake, impairing photosynthesis, and loss in production [21,22]. Extreme water stress conditions alter the physiological and morphological processes of the plant. The molecular and biochemical machinery is also affected under stress conditions. Reactive oxygen species (ROS), i.e., hydrogen peroxide, superoxide, singlet oxygen, and hydroxyl radical are excessively produced and accumulate in plant cells at water deficit conditions. Photoinhibition or photooxidation caused by ROS accumulation leads to uncontrolled photosynthetic components oxidation [23,24]. Water deficit/drought damages photosynthetic apparatus and photosynthetic pigments (chlorophyll $a$, chlorophyll $b$, and carotenoids) which also reduced the photosynthetic rate $[25,26]$. Photosynthetic pigments are linked to stress tolerance in plants $[27,28]$. Resistant cultivars had a higher amount of chlorophyll contents and carotenoids when exposed to water deficit conditions [29]. Water deficit conditions decrease contents of total chlorophyll $a$ and $b$ contents [30]. Water deficit conditions damage thylakoid lamella and reduce active oxygen species' chlorophyll contents [31]. The chlorophyll content is linked inversely with the severity and duration of water deficit conditions [32]. After oxidative stress, accumulation of proline acts as an adaptive stress response. The intracellular levels of proline can increase by $>100$-fold during stress [33]. Proline accumulation acts as a compatible osmolyte to buffer cytosolic $\mathrm{pH}$ and to balance cell redox status. Proline can also function as a molecular chaperone stabilizing the structure of proteins and as a ROS scavenger [24,34].

Plant anatomical structure is also disturbed when the plant is subjected to water deficit which affects the regulation of water through the vascular bundles. The effect of drought stress firstly occurs on the cell structure $[35,36]$. Increased hydraulic resistance and decreased growth are directly associated with xylem structure [37]. Anatomical changes in citrus rootstocks under abiotic stress influence their ability to survive. In drought 
conditions, the xylem vessel becomes emboli and dysfunctional. Therefore, plants with narrow and higher number of vessels are considered to be more drought tolerant [38]. The plant cells that face an environment with a shortage of water have generally shown an increase in vessel tissue, thick cell wall, reduction in cell size, and most severe condition cell wall and the cell membrane becomes ruptured $[39,40]$. The anatomical changes may occur to protect the plants under stress conditions. Multiple changes occur in response to water stress such as alteration of xylem phloem ratio, wall structure, lumen size, and lumen area that resist environmental stress on the plant [41]. The sensitivity of rootstock against drought stress is directly related to vessel dimension [42,43]. The vessel density of root and stem decreases with tree height as vessel diameter increases. The rootstock growth ability is dramatically affected by several xylem traits, xylem phloem ratio, vessel size, and vessel density $[43,44]$. Thus, traits play an important role in maintaining hydraulic conductance of root and stem and leaves $[37,45]$. Vessel number and size is the key factor to maintaining hydraulic conductance [46].

Strategies for drought tolerance are highly relevant in the case of rootstock selection and multiplication for ensuring continuous productivity. Rootstocks with a higher root growth ratio, better water use efficiency, higher root hydraulic conductivity, and lower osmatic conductance can withstand drought conditions while maintaining higher growth levels and mass accumulation $[47,48]$. After a severe drought, the recovery of tolerant rootstocks is much better than other rootstocks. Therefore, screening of drought-tolerant rootstocks is of utmost importance. For drought stress prevention, the plant increases water uptake efficiency either by increasing root density or deepening roots [26].

Climate changes shift the weather conditions by a high degree of unpredictability; water shortage in the soil and plant by a continuous increase in daily average temperature every year are inevitable, which intimidate overall globe agriculture industry stability by the negative effect on plant health and production consumer demand. Survival of the citrus industry against the water deficit needs to evaluate a tolerant/resistant citrus rootstock against the climate change scenario. The objective of the current study was to evaluate 10 citrus rootstocks against drought, based on visual changes, water potential, morphological and biochemical characters. After initial screening, the two most sensitive and tolerant genotypes were used to study the anatomical differences in leaf and stem to elucidate the drought tolerance mechanism in tolerant genotypes.

\section{Materials and Methods}

\subsection{Plant Materials, Experimental Site, and Growth Conditions}

Six months old citrus rootstocks potted plants were taken as experimental material. Ten genetically diverse citrus rootstocks were examined (details are given in Table 1). A potted plant experiment was executed at the Institute of Horticultural Science, University of Agriculture Faisalabad, Pakistan. Seeds of selected rootstock were taken from citrus rootstocks progeny block, HIS, UAF, Pakistan.

Table 1. Characteristics of citrus rootstocks used in this study.

\begin{tabular}{|c|c|c|c|c|}
\hline Rootstock & Botanical Name & Citrus Category & Leaf Shape & Parentage/Origin \\
\hline Gabbuchini & Citrus aurantium L. & Sour orange hybrid & Unifoliate & $\begin{array}{l}\text { C. aurantium } \\
\text { 'Bittersweet' } \times \\
\text { C. sinensis }\end{array}$ \\
\hline Gada dahi & $\begin{array}{c}\text { Citrus maxima Burm. Merrill/ } \\
\text { Citrus grandis L. Osbeck/ } \\
\text { Citrus decumana L. }\end{array}$ & Pummelo hybrid & Unifoliate & $\begin{array}{l}\text { Subcontinent } \\
\text { (Indo-Pak), seed } \\
\text { selection }\end{array}$ \\
\hline Sour orange & Citrus aurantium L. & Sour orange & Unifoliate & $\begin{array}{c}\text { Subcontinent } \\
\text { (Indo-Pak) }\end{array}$ \\
\hline Keen sour orange & Citrus aurantium L. & Sour orange & Unifoliate & Selection/root sprout \\
\hline Brazilian sour orange & Citrus aurantium L. & Sour orange & Unifoliate & $\begin{array}{l}\text { Open-pollinated seed } \\
\text { selection }\end{array}$ \\
\hline Rough lemon & Citrus jambhiri & Lemon & Unifoliate & $\begin{array}{l}\text { Open-pollinated seed } \\
\text { selection }\end{array}$ \\
\hline Sunki $\times$ bentake, & Citrus spp. & Unknown & Trifoliate & $\begin{array}{c}\text { Citrus sunki } \times \text { bentake } \\
\text { hybrid }\end{array}$ \\
\hline
\end{tabular}


Table 1. Cont.

\begin{tabular}{|c|c|c|c|c|}
\hline Rootstock & Botanical Name & Citrus Category & Leaf Shape & Parentage/Origin \\
\hline$x 639$ & Citroncirus spp. & Mandarin $\times$ Poncirus & Trifoliate & $\begin{array}{c}\text { Cleopatra mandarin } \times \\
\text { Poncirus trifoliata } \\
\text { hybrid }\end{array}$ \\
\hline Kirrumakki nucellar & Citrus limonia Osbeck & Lime & Unifoliate & $\begin{array}{l}\text { Unknow/Subcontinent } \\
\text { (Indo-Pak) }\end{array}$ \\
\hline $\begin{array}{l}\text { Rangpur Poona } \\
\text { nucellar }\end{array}$ & Citrus limonia Osbeck & Lime & Unifoliate & $\begin{array}{l}\text { Unknow /Subcontinent } \\
\text { (Indo-Pak) }\end{array}$ \\
\hline
\end{tabular}

The seeds were sown in transparent grow bags (height $12^{\prime \prime}$ and width $6^{\prime \prime}$ ) and placed in a growth chamber (Model: BST/PGC-175; Bionics Scientific Technologies (p) ltd., Delhi, India) at $32 \pm 2{ }^{\circ} \mathrm{C}$, relative humidity oscillating between 80 and $95 \%$ and $12-14 \mathrm{~h}$ of light. Potted media contained sand, silt, and clay (1:1:1). These plants were placed in the chamber for six months before the treatment application and during growth, pots were regularly irrigated with tap water (75\% field capacity) and fertilized weekly with nutrient solution $\left(1.0 \mathrm{~g} \mathrm{~L}^{-1} \mathrm{Ca}\left(\mathrm{NO}_{3}\right)_{2}, 0.4 \mathrm{~g} \mathrm{~L}^{-1} \mathrm{KNO}_{3}, 0.6 \mathrm{~g} \mathrm{~L}^{-1} \mathrm{MgSO}_{4}\right.$ and $0.4 \mathrm{~g} \mathrm{~L}^{-1} \mathrm{NH}_{4} \mathrm{H}_{2} \mathrm{PO}_{4}$ (MAP)).

\subsection{Water Regimes and Treatments}

Citrus rootstocks were subjected to water deficit treatment applications after six months of growth in the controlled growth condition. Plants were exposed to three different groups: controlled condition, moderate water deficit (moderate drought), and severe water deficit (severe drought). Each treatment group consists of 10 citrus rootstocks with three replications. Control plants (sufficient water and optimal temperature $\sim 32{ }^{\circ} \mathrm{C}$ ) were irrigated once every two days (75\% field capacity). Water deficit treatments were moderate (50\% field capacity) and severe ( $25 \%$ field capacity) deficit conditions. Water was applied with 2 days interval for control and treated plants. The controlled and water deficit exposed genotypes were kept at $32 \pm 2{ }^{\circ} \mathrm{C}$ for day and night temperature in a controlled growth room. Thereby, three experimental groups were established as control, moderate water deficit, and severe water deficit conditions.

\subsection{Morphological and Biomass Measurements}

Leaves were visually observed for leaf necrosis or chlorosis on rootstock seedlings after 15 days of stress applications. Plant height $(\mathrm{cm})$ and root length $(\mathrm{cm})$ were measured with help of a scale after uprooting the plants at the end of the experiment (15 DAS). Leaf water potential was determined to collect the healthy leaf samples at dawn (Shafqat et al., 2021) by using a pressure chamber (Model 3000, Soil moisture Equipment, Santa Barbara, CA, USA). Root and shoot fresh biomass were weighed on electric balance (TS-200 Digital Electronic Scale), oven-dried $\left(70{ }^{\circ} \mathrm{C}\right)$ for $48 \mathrm{~h}$, and weighed again separately [48]. Shoot and root moisture content were calculated using Equation (1):

$$
\text { Moisture content }(\%)=\frac{\text { Fresh weight }- \text { dry weight }}{\text { Fresh weight }} \times 100
$$

\subsection{Biochemical and Stress-Associated Biomarker Measurements}

\subsubsection{Leaf Photosynthetic Pigments Contents}

Leaf sample of $0.5 \mathrm{~g}$ was cut into small pieces and homogenized by adding $80 \%$ acetone (v/v). The extract was transferred to a $15 \mathrm{~mL}$ tube [48]. Test tubes were placed in the dark to avoid light for $24 \mathrm{~h}$ and filtered through filter paper. The absorbance was determined at $663 \mathrm{~nm}$ for chlorophyll a, $647 \mathrm{~nm}$ for chlorophyll b, and $470 \mathrm{~nm}$ for carotenoid. The Chl $a$, $\mathrm{Chl} b$, and carotenoids contents were determined using Equations (2)-(4), respectively.

$$
\mathrm{Chl} a(\mathrm{mg} / \mathrm{g} \text { fresh weight })=\frac{(12.7 \mathrm{OD} 663-2.69 \mathrm{OD} 645) \times \mathrm{V}}{1000 \times \mathrm{W}}
$$




$$
\begin{gathered}
\text { Chl } b(\mathrm{mg} / \mathrm{g} \text { fresh weight })=\frac{(22.9 \mathrm{OD} 645-4.68 \mathrm{OD} 663) \times \mathrm{V}}{1000 \times \mathrm{W}} \\
\text { Carotenoids }(\mathrm{mg} / \mathrm{g} \text { fresh weight })=\frac{\text { O.D } 480+0.114(\mathrm{O} . \mathrm{D} 663)-0.638(\text { O.D } 645)}{\mathrm{Em} \times 100}
\end{gathered}
$$

where $\mathrm{V}=$ volume of the sample, $\mathrm{W}=$ weight of fresh tissue, $\mathrm{Em}=2500$.

\subsubsection{Determination of Proline}

Proline contents were assessed by following the acid ninhydrin method [49]. Fresh $0.5 \mathrm{~g}$ leaf material was extracted using $10 \mathrm{~mL}$ of $3 \%$ sulfosalicylic acid (Panreac, Barcelona, Spain) for $30 \mathrm{~min}$. Centrifugation at $2000 \mathrm{~g}$ for $20 \mathrm{~min}$ at $4{ }^{\circ} \mathrm{C}$ was done. The $2 \mathrm{~mL}$ of filtered aqueous extract was mixed with acid ninhydrin reagent $(2 \mathrm{~mL})$, and glacial acetic acid $(2 \mathrm{~mL})$, and heated at $100^{\circ} \mathrm{C}$ for $1 \mathrm{~h}$. The reaction mixture after cooling was segregated against toluene $(4 \mathrm{~mL})$ and the absorbance of the organic phase was recorded at $520 \mathrm{~nm}$ using a spectrophotometer (IRMECO UV/VIS Model U2020). The resulting values were related with a standard curve plotted using known amounts of proline (Sigma, St Louis, MO, USA).

\subsubsection{Determination of Hydrogen Peroxide $\left(\mathrm{H}_{2} \mathrm{O}_{2}\right)$}

The leaf tissues of $0.1 \mathrm{~g}$ were ground in $1 \mathrm{~mL}$ of trichloroacetic acid solution $(0.1 \%)$ within an ice bath [50]. After preparation in Eppendorf, the samples were centrifuged at $9719 \times g$ for $15 \mathrm{~min}$. The supernatant of $500 \mu \mathrm{L}$ was transferred into a new tube having a mixture of $1 \mathrm{M} \mathrm{KI}(1000 \mu \mathrm{L})$ and $10 \mathrm{mM}$ potassium phosphate buffer $(500 \mu \mathrm{L})$. Absorbance was read at $390 \mathrm{~nm}$ in a UV-1900 spectrophotometer (Eppendorf BioSpectrometer ${ }^{\circledR}$ basic). $\mathrm{H}_{2} \mathrm{O}_{2}$ content was calculated as $\mu \mathrm{mol} \mathrm{g}^{-1}$ DW by comparing the absorbance values against the standard curve of commercial $\mathrm{H}_{2} \mathrm{O}_{2}$.

\subsection{Stem and Leaf Anatomical Evaluation}

\subsubsection{Plant Material and Experiment}

Two highly tolerant (Brazilian sour orange and Gadha dahi) and highly sensitive (Rangpur Poona nucellar and Sunki $\times$ bentake) genotypes from the first screening study were selected to screen based on the leaf and stem anatomical study. These selected rootstocks were exposed to the same treatments for 15 days then leaf and stem samples were collected for anatomical studies.

\subsubsection{Preservation, Sectioning, Staining, and Mounting}

Stem and leaf samples $(2 \mathrm{~cm})$ were preserved in a formalin acetic alcohol (FAA) solution containing $5 \%$ formalin, $10 \%$ acetic acid, $50 \%$ ethanol, and $35 \%$ distilled water. Thereafter, the preserved material was subsequently transferred to an acetic alcohol solution (acetic acid 25\% v/v, ethanol 75\%) for the long-term preservation of samples. A free-hand sectioning technique was used to prepare permanent slides of stem and leaf transverse sections cut with the razor blade, and some fine sections were carefully picked up on wash glass for staining and dehydration through a series of washings with ethanol $(30 \%$, $50 \%$, and then $70 \%$ for 15 min each). For staining, the lignified tissues (xylem vessels and sclerenchyma) were transferred to safranin ( $1.0 \mathrm{~g}$ dissolved in $100 \mathrm{~mL}, 70 \%$ alcohol) for $20 \mathrm{~min}$, dehydrated in $90 \%$ alcohol for $5 \mathrm{~min}$, and stained with fast green (1.0 g dissolved in $90 \%$ ethanol) for one minute. Finally, the tissues were washed three times with absolute alcohol and then transferred to xylene for cleaning the contrast. The sections were mounted in Canada balsam by putting a drop of resin on a slide and placing the sections on the slides and photographed with a digital camera attached to a compound microscope.

\subsubsection{Anatomical Traits}

The stem and leaf cross-sectional area $\left(\mathrm{mm}^{2}\right)$ was measured under a compound light microscope (Olympus MX63, Japan) by recording the maximum length and width of the root sections. Cells present in plants epidermis were measured with the help of ruler 
or $\mathrm{cm}$ scales, and the length of the epidermal cell was multiplied by 41.5 to obtain a value in micrometers. Vascular bundle length was calculated by measuring xylem and phloem which were multiplied by 41.5 to obtain the value in $\mu \mathrm{m}$. Xylem thickness was calculated by the width of the xylem region. Metaxylem area, phloem cell area, pith cell area, and the cortical area were calculated in $\mu \mathrm{m}^{2}$ by measuring the length and width of each trait. Cortical thickness was obtained by measuring the total width of the cortical region in micrometers. By using Equation (5), the areas of the different cells and tissues were calculated (which was modified from the area of the circle).

$$
\text { Area }=\frac{\text { Maximum length } \times \text { Maximum width }}{28} \times 22
$$

\subsection{Statistical Analysis}

Throughout this study, all experiments were laid out using a full factorial split-plot design arranged in randomized complete blocks using rootstocks as main plots and water regimes in the subplots. All experiments were carried out using at least three biological replicates for each treatment. The analysis of variance (ANOVA) was used to test the significant differences among different drought levels ( $\left.p_{\text {Drought }}\right)$, rootstocks $\left(p_{\text {Rootstocks }}\right)$, and their interaction $\left(p_{\text {Drought }} \times\right.$ rootstocks $)$. Tukey's honestly significant difference (HSD) test was used for post-hoc analysis ( $p_{\text {Drought }} \times$ rootstocks $<0.05$ ). Moreover, the data matrix of all dependent variables was used to perform the principal component analysis (PCA). Finally, means of all dependent variables were used for two-way hierarchical cluster analysis (HCA). Similarities and variations between treatments were presented as a heat map.

\section{Results}

\subsection{Drought Negatively Affects the Roots and Shoots Length}

Both root and shoot length were significantly affected by varying drought levels $\left(p_{\text {Drought }}<0.0001\right)$ and rootstocks $\left(p_{\text {Rootstock }}<0.0001\right)$ (Table 2$)$. Briefly, the root length of different citrus rootstocks was significantly reduced when plants were stressed with different levels of drought. Although control Brazilian sour orange had the highest root length $\left(27.67 \pm 0.58 \mathrm{~cm} ; p_{\text {Drought }} \times\right.$ Rootstock $\left.<0.0001\right)$, shortest root lengths were recorded by Sunki $\times$ bentake when stressed with moderate $(10.33 \pm 0.58 \mathrm{~cm})$ or severe drought $(9.33 \pm 0.58 \mathrm{~cm})$ and Rangpur Poona nucellar rootstock under severe drought conditions $(10.33 \pm 0.58 \mathrm{~cm})($ Table 2). On the other hand, while there were no significant differences in shoot length of all tested rootstock, except Gabbuchini, under normal growth conditions, shoot lengths were significantly reduced under various drought levels. Rangpur Poona nucellar rootstock had the shortest shoots $(5.33 \pm 0.58 \mathrm{~cm})$, followed by Sunki $\times$ bentake $(6.00 \pm 1.00 \mathrm{~cm})$ when stressed under severe drought $\left(p_{\text {Drought }} \times\right.$ Rootstock $\left.<0.0001\right)$ (Table 2).

\subsection{Drought Stress Disrupts the Water Relations of Citrus Rootstocks}

As strong wilt phenotype was visually observed on citrus seedlings after 15 days post applications (DPA; Figure 1), the water relations (water potential, root moisture content, and shoot moisture content) were assessed (Table 2). For instance, water potential was reduced significantly by water-deficient $\left(p_{\text {Drought }}<0.0001\right)$ and rootstocks $\left(p_{\text {Rootstock }}=0.0018\right)$. Kirrumakki nucellar rootstock had the highest water potential $(-0.11 \pm 0.66 \mathrm{Mpa})$, whereas, Keen sour orange, Rough lemon, Brazilian sour orange, Sunki $\times$ bentake, X639, Kirrumakki nucellar, and Rangpur Poona nucellar rootstocks had the lowest water potential when severely stressed with water deficiency, without significant differences between them $\left(p_{\text {Drought }} \times\right.$ Rootstock $=$ 0.0250; Table 2). Likewise, the moisture content of both root and shoot was significantly reduced by drought levels $\left(p_{\text {Drought }}<0.0001\right)$ and rootstocks $\left(p_{\text {Rootstock }}<0.0001\right)$ (Table 2$)$. Normal irrigated Brazilian sour orange had the highest moisture content of root $(75.67 \pm 0.59 \%)$ and shoot $(84.75 \pm 0.65 \%)$, whereas Rangpur Poona nucellar rootstock had the lowest moisture content of root $(36.33 \pm 0.58 \%)$ and shoot $(40.69 \pm 0.65 \%)$ under severe drought conditions (Table 2). 
Table 2. Effect of different water regimes on the root length $(\mathrm{cm})$, shoot length $(\mathrm{cm})$, and water relations of 10 differentcitrus rootstocks with distinct degrees of tolerance to drought stress.

\begin{tabular}{|c|c|c|c|c|c|c|}
\hline & \multirow[b]{2}{*}{ Rootstock } & \multirow[b]{2}{*}{$\begin{array}{l}\text { Root Length } \\
\text { (cm) }\end{array}$} & \multirow[b]{2}{*}{$\begin{array}{l}\text { Shoot Length } \\
(\mathrm{cm})\end{array}$} & \multirow{2}{*}{$\begin{array}{l}\text { Water Potential } \\
\text { (Mpa) }\end{array}$} & \multicolumn{2}{|c|}{ Moisture Content } \\
\hline & & & & & $\underset{(\%)}{\text { Root }}$ & $\begin{array}{c}\text { Shoot } \\
(\%)\end{array}$ \\
\hline \multirow{10}{*}{ 它 } & Gabbuchini & $25.33 \pm 0.58 \mathrm{ab}$ & $21.00 \pm 1.00 \mathrm{~b}$ & $-0.32 \pm 0.02 \mathrm{a}-\mathrm{e}$ & $66.00 \pm 1.00 \mathrm{cde}$ & $73.92 \pm 1.12$ \\
\hline & Gada dahi & $24.33 \pm 0.58 \mathrm{bc}$ & $24.33 \pm 0.58 \mathrm{a}$ & $-0.23 \pm 0.02 \mathrm{a}-\mathrm{d}$ & $69.00 \pm 1.00 \mathrm{~b}$ & $77.28 \pm 1.12 \mathrm{~b}$ \\
\hline & Sour orange & $24.33 \pm 0.58 \mathrm{bc}$ & $23.67 \pm 0.58 \mathrm{a}$ & $-0.27 \pm 0.06 \mathrm{a}-\mathrm{e}$ & $64.67 \pm 0.58 \mathrm{e}$ & $72.43 \pm 0.65 \mathrm{e}$ \\
\hline & Keen sour orange & $25.33 \pm 0.58 \mathrm{ab}$ & $25.33 \pm 0.58 \mathrm{a}$ & $-0.19 \pm 0.02 \mathrm{abc}$ & $67.33 \pm 0.58 \mathrm{bcd}$ & $75.41 \pm 0.65 \mathrm{bcd}$ \\
\hline & Rough lemon & $26.00 \pm 1.00 \mathrm{ab}$ & $25.00 \pm 1.00 \mathrm{a}$ & $-0.19 \pm 0.03 \mathrm{abc}$ & $64.67 \pm 0.58 \mathrm{e}$ & $72.43 \pm 0.65 \mathrm{e}$ \\
\hline & Brazilian sour orange & $27.67 \pm 0.58 \mathrm{a}$ & $25.00 \pm 1.00 \mathrm{a}$ & $-0.23 \pm 0.02 \mathrm{a}-\mathrm{d}$ & $75.67 \pm 0.58 \mathrm{a}$ & $84.75 \pm 0.65 \mathrm{a}$ \\
\hline & Sunki $\times$ bentake & $12.33 \pm 0.58 \mathrm{jkl}$ & $24.67 \pm 0.58 \mathrm{a}$ & $-0.28 \pm 0.05 \mathrm{a}-\mathrm{e}$ & $65.33 \pm 0.58 \mathrm{de}$ & $73.17 \pm 0.65 \mathrm{de}$ \\
\hline & X639 & $24.33 \pm 0.58 \mathrm{bc}$ & $24.33 \pm 0.58 \mathrm{a}$ & $-0.26 \pm 0.06 \mathrm{a}-\mathrm{e}$ & $67.00 \pm 1.00 \mathrm{~b}-\mathrm{e}$ & $75.04 \pm 1.12 \mathrm{~b}-\mathrm{e}$ \\
\hline & Kirrumakki nucellar & $24.00 \pm 1.00 \mathrm{bc}$ & $24.00 \pm 1.00 \mathrm{a}$ & $-0.23 \pm 0.02 \mathrm{a}-\mathrm{d}$ & $68.00 \pm 1.00 \mathrm{bc}$ & $76.16 \pm 1.12 \mathrm{bc}$ \\
\hline & Rangpur poona nucellar & $24.67 \pm 0.58 \mathrm{bc}$ & $24.67 \pm 0.58 \mathrm{a}$ & $-0.23 \pm 0.02 \mathrm{a}-\mathrm{d}$ & $66.00 \pm 1.00 \mathrm{cde}$ & $73.92 \pm 1.12$ cde \\
\hline \multirow{10}{*}{ 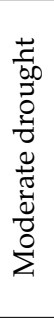 } & Gabbuchini & $15.67 \pm 0.58 \mathrm{ghi}$ & $12.00 \pm 1.00 \mathrm{ghi}$ & $-0.60 \pm 0.05 \mathrm{a}-\mathrm{f}$ & $55.67 \pm 0.58 \mathrm{fg}$ & $62.35 \pm 0.65 \mathrm{fg}$ \\
\hline & Gada dahi & $22.67 \pm 0.58 \mathrm{~cd}$ & $18.33 \pm 0.58 \mathrm{c}$ & $-0.15 \pm 0.58 \mathrm{ab}$ & $64.67 \pm 0.58 \mathrm{e}$ & $72.43 \pm 0.65 \mathrm{e}$ \\
\hline & Sour orange & $19.00 \pm 1.00 \mathrm{ef}$ & $15.67 \pm 0.58 \mathrm{de}$ & $-0.61 \pm 0.06 \mathrm{a}-\mathrm{f}$ & $54.00 \pm 1.00 \mathrm{~g}$ & $60.48 \pm 1.12 \mathrm{~g}$ \\
\hline & Keen sour orange & $21.00 \pm 1.00 \mathrm{de}$ & $18.33 \pm 0.58 \mathrm{c}$ & $-0.62 \pm 0.14 \mathrm{a}-\mathrm{f}$ & $58.00 \pm 1.00 \mathrm{f}$ & $64.96 \pm 1.12 \mathrm{f}$ \\
\hline & Rough lemon & $17.33 \pm 0.58 \mathrm{fg}$ & $14.33 \pm 0.58 \mathrm{efg}$ & $-0.68 \pm 0.03 \mathrm{~b}-\mathrm{f}$ & $54.33 \pm 0.58 \mathrm{~g}$ & $60.85 \pm 0.65 \mathrm{~g}$ \\
\hline & Brazilian sour orange & $25.67 \pm 0.58 \mathrm{ab}$ & $19.00 \pm 1.00 \mathrm{bc}$ & $-0.70 \pm 0.03 \mathrm{c}-\mathrm{f}$ & $65.00 \pm 1.00 \mathrm{de}$ & $72.80 \pm 1.12 \mathrm{de}$ \\
\hline & Sunki $\times$ bentake & $10.33 \pm 0.58 \mathrm{~lm}$ & $14.67 \pm 0.58 \mathrm{def}$ & $-0.66 \pm 0.04 \mathrm{~b}-\mathrm{f}$ & $50.33 \pm 0.58 \mathrm{~h}$ & $56.37 \pm 0.65 \mathrm{~h}$ \\
\hline & X639 & $16.33 \pm 1.53 \mathrm{gh}$ & $13.00 \pm 1.00 \mathrm{fgh}$ & $-0.74 \pm 0.06 \mathrm{def}$ & $54.33 \pm 0.58 \mathrm{~g}$ & $60.85 \pm 0.65 \mathrm{~g}$ \\
\hline & Kirrumakki nucellar & $15.33 \pm 0.58$ ghi & $12.00 \pm 1.00 \mathrm{ghi}$ & $-0.11 \pm 0.66 \mathrm{a}$ & $54.00 \pm 1.00 \mathrm{~g}$ & $60.48 \pm 1.12 \mathrm{~g}$ \\
\hline & Rangpur poona nucellar & $14.67 \pm 0.58 \mathrm{hij}$ & $11.00 \pm 1.00 \mathrm{hij}$ & $-0.78 \pm 0.03 \mathrm{efg}$ & $48.67 \pm 0.58 \mathrm{hi}$ & $54.51 \pm 0.65 \mathrm{hi}$ \\
\hline \multirow{10}{*}{ 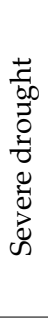 } & Gabbuchini & $11.67 \pm 1.15 \mathrm{klm}$ & $08.33 \pm 0.58 \mathrm{kl}$ & $-1.30 \pm 0.06 \mathrm{gh}$ & $46.33 \pm 0.58 \mathrm{ij}$ & $51.89 \pm 0.65 \mathrm{ij}$ \\
\hline & Gada dahi & $19.33 \pm 0.58$ ef & $14.67 \pm 0.58 \mathrm{def}$ & $-1.14 \pm 0.01 \mathrm{fgh}$ & $54.67 \pm 0.58 \mathrm{~g}$ & $61.23 \pm 0.65 \mathrm{~g}$ \\
\hline & Sour orange & $14.67 \pm 0.58 \mathrm{hij}$ & $11.67 \pm 0.58 \mathrm{hi}$ & $-1.30 \pm 0.05 \mathrm{gh}$ & $46.67 \pm 0.58 \mathrm{ij}$ & $52.27 \pm 0.65 \mathrm{ij}$ \\
\hline & Keen sour orange & $17.33 \pm 0.58 \mathrm{fg}$ & $13.00 \pm 1.00 \mathrm{fgh}$ & $-1.39 \pm 0.07 \mathrm{~h}$ & $50.00 \pm 1.00 \mathrm{~h}$ & $56.00 \pm 1.12 \mathrm{~h}$ \\
\hline & Rough lemon & $13.67 \pm 0.58 \mathrm{ijk}$ & $10.33 \pm 0.58 \mathrm{ijk}$ & $-1.39 \pm 0.04 \mathrm{~h}$ & $45.00 \pm 1.00 \mathrm{jk}$ & $50.40 \pm 1.12 \mathrm{jk}$ \\
\hline & Brazilian sour orange & $24.33 \pm 0.58 \mathrm{bc}$ & $17.00 \pm 1.00 \mathrm{~cd}$ & $-1.57 \pm 0.03 \mathrm{~h}$ & $57.67 \pm 1.53 \mathrm{f}$ & $64.59 \pm 1.71 \mathrm{f}$ \\
\hline & Sunki $\times$ bentake & $09.33 \pm 0.58 \mathrm{~lm}$ & $06.00 \pm 1.00 \mathrm{~lm}$ & $-1.39 \pm 0.03 \mathrm{~h}$ & $39.67 \pm 0.581$ & $44.43 \pm 0.651$ \\
\hline & X639 & $14.00 \pm 1.00$ hijk & $08.33 \pm 0.58 \mathrm{kl}$ & $-1.36 \pm 0.06 \mathrm{~h}$ & $43.33 \pm 0.58 \mathrm{k}$ & $48.53 \pm 0.65 \mathrm{k}$ \\
\hline & Kirrumakki nucellar & $13.33 \pm 0.58 \mathrm{ijk}$ & $08.67 \pm 0.58 \mathrm{jk}$ & $-1.38 \pm 0.03 \mathrm{~h}$ & $45.00 \pm 1.00 \mathrm{jk}$ & $50.40 \pm 1.12 \mathrm{jk}$ \\
\hline & Rangpur poona nucellar & $10.33 \pm 0.58 \mathrm{~lm}$ & $05.33 \pm 0.58 \mathrm{~m}$ & $-1.40 \pm 0.03 \mathrm{~h}$ & $36.33 \pm 0.58 \mathrm{~m}$ & $40.69 \pm 0.65 \mathrm{~m}$ \\
\hline \multicolumn{7}{|c|}{$p$-value } \\
\hline & $p_{\text {Drought }}$ & $<0.0001$ & $<0.0001$ & $<0.0001$ & $<0.0001$ & $<0.0001$ \\
\hline & $p_{\text {Rootstock }}$ & $<0.0001$ & $<0.0001$ & $=0.0018$ & $<0.0001$ & $<0.0001$ \\
\hline & $p_{\text {Drought }} \times$ Rootstock & $<0.0001$ & $<0.0001$ & $=0.0250$ & $<0.0001$ & $<0.0001$ \\
\hline
\end{tabular}

Data presented are means \pm standard deviation (mean \pm SD) of three replicates. Different letters indicate statistically significant differences among treatments, while "ns" signifies no significant differences between them according to Tukey's honestly significant difference test $(p<0.05)$.
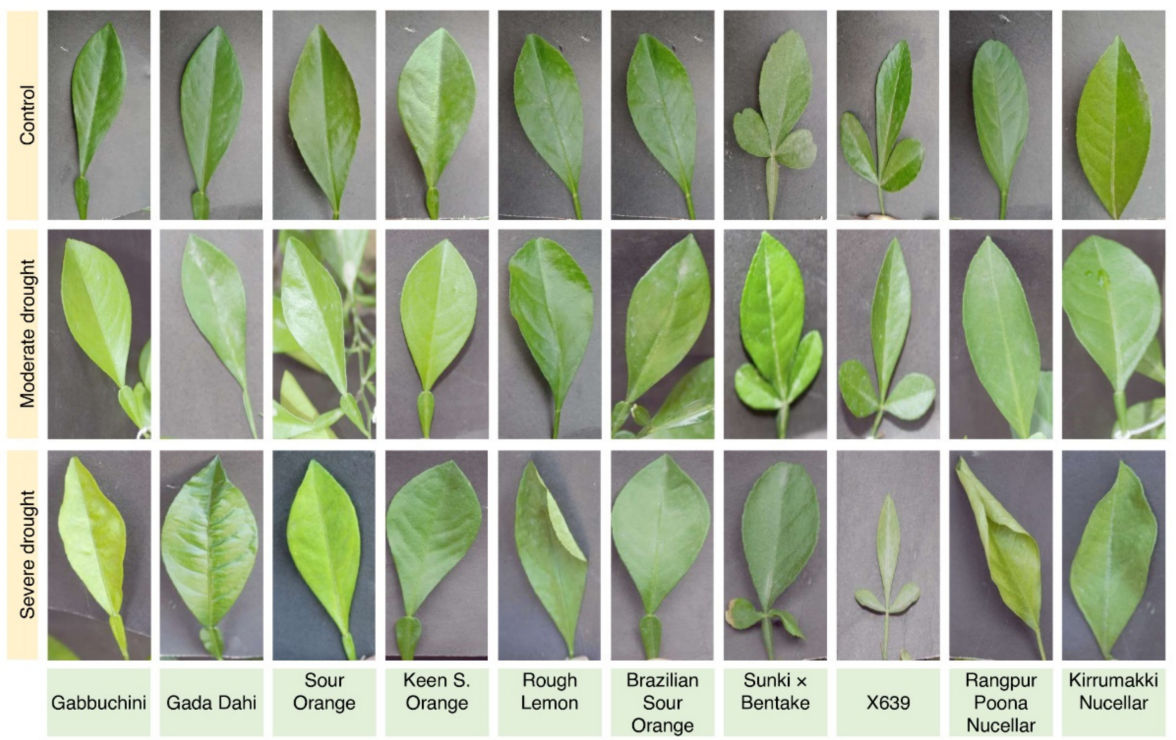

Figure 1. Effect of different water regimes on the leaf phenotype of 10 different citrus rootstocks with distinct degrees of tolerance to drought stress. 


\subsection{Water Deficiency Interrupts the Photosynthetic Pigments of Citrus Rootstocks}

Drought stress considerably lessened chlorophyll $a$, chlorophyll $b$, and carotenoids content of different rootstocks ( $p_{\text {Drought }}<0.0001$; Table 3 ). Normal irrigated Brazilian sour orange had the highest chlorophyll $a$ content $\left(3.74 \pm 0.12 \mathrm{mg} \mathrm{g}^{-1} \mathrm{FW}\right)$, while Rangpur Poona nucellar rootstock had the lowest chlorophyll $a$ content $\left(1.57 \pm 0.04 \% \mathrm{mg} \mathrm{g}^{-1} \mathrm{FW}\right.$; $p_{\text {Drought }} \times$ Rootstock $\left.<0.0001\right)$. Furthermore, there was no significant difference in chlorophyll $b$ content among all tested rootstocks, except Gabbuchini and Gada dahi, under normal irrigation conditions. However, chlorophyll $b$ content was significantly decreased under water deficiency conditions (Table 3). Likewise, there were no significant differences in carotenoids content of all studied citrus rootstocks, except Gabbuchini, under normal irrigation conditions. Nevertheless, carotenoids content was significantly reduced when citrus rootstocks were stressed with different drought levels. It is worth mentioning that Rangpur Poona nucellar rootstock had the lowest carotenoids content $\left(0.14 \pm 0.03 \mathrm{mg} \mathrm{g}^{-1} \mathrm{FW}\right)$, followed by Sunki $\times$ bentake $\left(0.23 \pm 0.02 \mathrm{mg} \mathrm{g}^{-1} \mathrm{FW}\right)$ under severe drought conditions (Table 3).

Table 3. Effect of different water regimes on the photosynthetic pigments, $\mathrm{H}_{2} \mathrm{O}_{2}$, and proline content of 10 different citrus rootstocks with distinct degrees of tolerance to drought stress.

\begin{tabular}{|c|c|c|c|c|c|c|}
\hline & Rootstock & $\begin{array}{l}\text { Chlorophyll } a \\
\left(\mathrm{mg} \mathrm{g}^{-1} \mathrm{FW}\right)\end{array}$ & $\begin{array}{l}\text { Chlorophyll } b \\
\left(\mathrm{mg} \mathrm{g}^{-1} \mathrm{FW}\right)\end{array}$ & $\begin{array}{l}\text { Carotenoids } \\
\left(\mathrm{mg} \mathrm{g}^{-1} \mathrm{FW}\right)\end{array}$ & $\underset{\left(\mu \mathrm{mol} \mathrm{g} \mathrm{g}^{-1} \mathrm{FW}\right)}{\mathrm{H}_{2} \mathrm{O}_{2}}$ & $\begin{array}{c}\text { Proline } \\
\left(\mu \mathrm{mol} \mathrm{g}{ }^{-1} \mathrm{FW}\right)\end{array}$ \\
\hline \multirow{10}{*}{$\begin{array}{l}\bar{O} \\
\stackrel{+}{*} \\
0 \\
0\end{array}$} & Gabbuchini & $3.52 \pm 0.03 \mathrm{~b}$ & $1.43 \pm 0.06 \mathrm{abc}$ & $1.05 \pm 0.04 \mathrm{ab}$ & $43.00 \pm 2.65 \mathrm{~d}$ & $0.33 \pm 0.03 c-h$ \\
\hline & Gada dahi & $3.54 \pm 0.01 \mathrm{ab}$ & $1.47 \pm 0.06 \mathrm{ab}$ & $1.13 \pm 0.04 \mathrm{a}$ & $44.33 \pm 5.03 \mathrm{~d}$ & $0.35 \pm 0.01 \mathrm{c}-\mathrm{g}$ \\
\hline & Sour orange & $3.53 \pm 0.02 \mathrm{~b}$ & $1.53 \pm 0.06 \mathrm{a}$ & $1.18 \pm 0.04 \mathrm{a}$ & $42.33 \pm 1.15 \mathrm{~d}$ & $0.38 \pm 0.02 \mathrm{c}-\mathrm{f}$ \\
\hline & Keen sour orange & $3.57 \pm 0.05 \mathrm{ab}$ & $1.50 \pm 0.10 \mathrm{a}$ & $1.15 \pm 0.08 \mathrm{a}$ & $46.00 \pm 2.00 \mathrm{~d}$ & $0.29 \pm 0.00 \mathrm{fgh}$ \\
\hline & Rough lemon & $3.60 \pm 0.08 \mathrm{ab}$ & $1.50 \pm 0.10 \mathrm{a}$ & $1.15 \pm 0.08 \mathrm{a}$ & $45.33 \pm 2.52 \mathrm{~d}$ & $0.32 \pm 0.03 \mathrm{c}-\mathrm{h}$ \\
\hline & Brazilian sour orange & $3.74 \pm 0.12 \mathrm{a}$ & $1.57 \pm 0.06 \mathrm{a}$ & $1.26 \pm 0.12 \mathrm{a}$ & $45.00 \pm 2.65 \mathrm{~d}$ & $0.38 \pm 0.02 \mathrm{c}-\mathrm{f}$ \\
\hline & Sunki $\times$ bentake & $3.50 \pm 0.05 b$ & $1.53 \pm 0.06 \mathrm{a}$ & $1.18 \pm 0.04 \mathrm{a}$ & $44.67 \pm 0.58 \mathrm{~d}$ & $0.25 \pm 0.01 \mathrm{ghi}$ \\
\hline & X639 & $3.51 \pm 0.06 \mathrm{~b}$ & $1.57 \pm 0.06 \mathrm{a}$ & $1.21 \pm 0.04 \mathrm{a}$ & $39.67 \pm 6.03 \mathrm{~d}$ & $0.26 \pm 0.00 \mathrm{ghi}$ \\
\hline & Kirrumakki nucellar & $3.57 \pm 0.08 \mathrm{ab}$ & $1.57 \pm 0.06 \mathrm{a}$ & $1.26 \pm 0.04 \mathrm{a}$ & $42.67 \pm 1.53 \mathrm{~d}$ & $0.41 \pm 0.01 \mathrm{bcd}$ \\
\hline & Rangpur poona nucellar & $3.68 \pm 0.04 \mathrm{ab}$ & $1.53 \pm 0.06 \mathrm{a}$ & $1.18 \pm 0.04 \mathrm{a}$ & $44.67 \pm 2.08 \mathrm{~d}$ & $0.17 \pm 0.02 \mathrm{i}-1$ \\
\hline \multirow{10}{*}{ 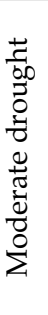 } & Gabbuchini & $2.95 \pm 0.04 \mathrm{de}$ & $1.23 \pm 0.06 \mathrm{cde}$ & $0.77 \pm 0.08 \mathrm{c}-\mathrm{f}$ & $64.33 \pm 2.08 c$ & $0.18 \pm 0.03 \mathrm{i}-1$ \\
\hline & Gada dahi & $3.10 \pm 0.01$ cde & $1.27 \pm 0.06 \mathrm{bcd}$ & $0.85 \pm 0.05 \mathrm{bcd}$ & $68.33 \pm 3.06 \mathrm{bc}$ & $0.29 \pm 0.01 \mathrm{fgh}$ \\
\hline & Sour orange & $2.99 \pm 0.04$ cde & $1.03 \pm 0.12 \mathrm{efg}$ & $0.79 \pm 0.09 \mathrm{cde}$ & $64.67 \pm 1.53 c$ & $0.26 \pm 0.04 \mathrm{ghi}$ \\
\hline & Keen sour orange & $3.12 \pm 0.02$ cde & $1.23 \pm 0.06 \mathrm{cde}$ & $0.82 \pm 0.12$ cde & $62.67 \pm 2.08 c$ & $0.23 \pm 0.06 \mathrm{hij}$ \\
\hline & Rough lemon & $3.14 \pm 0.03 \mathrm{~cd}$ & $0.93 \pm 0.15 \mathrm{fgh}$ & $0.72 \pm 0.12 \mathrm{c}-\mathrm{f}$ & $62.00 \pm 1.00 \mathrm{c}$ & $0.25 \pm 0.05 \mathrm{ghi}$ \\
\hline & Brazilian sour orange & $3.19 \pm 0.04 \mathrm{c}$ & $1.37 \pm 0.06 \mathrm{abc}$ & $0.87 \pm 0.04 \mathrm{bcd}$ & $66.00 \pm 2.65 c$ & $0.31 \pm 0.02 \mathrm{~d}-\mathrm{h}$ \\
\hline & Sunki $\times$ bentake & $2.66 \pm 0.05 \mathrm{~g}$ & $0.80 \pm 0.10 \mathrm{hi}$ & $0.61 \pm 0.05 \mathrm{e}-\mathrm{h}$ & $66.00 \pm 1.73 c$ & $0.14 \pm 0.05 \mathrm{jkl}$ \\
\hline & X639 & $3.11 \pm 0.03$ cde & $1.13 \pm 0.06 \mathrm{def}$ & $0.79 \pm 0.09$ cde & $60.67 \pm 1.53 c$ & $0.25 \pm 0.02 \mathrm{ghi}$ \\
\hline & Kirrumakki nucellar & $2.92 \pm 0.03$ ef & $1.13 \pm 0.06 \mathrm{def}$ & $0.90 \pm 0.12 \mathrm{bc}$ & $64.67 \pm 1.53 c$ & $0.41 \pm 0.00 \mathrm{bcd}$ \\
\hline & Rangpur poona nucellar & $2.54 \pm 0.02 \mathrm{gh}$ & $0.73 \pm 0.06 \mathrm{~h}-\mathrm{k}$ & $0.57 \pm 0.07 \mathrm{f}-\mathrm{i}$ & $64.67 \pm 2.52 c$ & $0.08 \pm 0.061$ \\
\hline \multirow{14}{*}{ 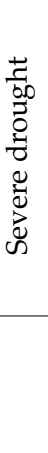 } & Gabbuchini & $2.20 \pm 0.03 \mathrm{jk}$ & $0.77 \pm 0.06 \mathrm{hij}$ & $0.44 \pm 0.04 \mathrm{c}-\mathrm{f}$ & $83.00 \pm 2.00 \mathrm{a}$ & $0.41 \pm 0.01 \mathrm{abc}$ \\
\hline & Gada dahi & $2.45 \pm 0.04 \mathrm{hi}$ & $0.83 \pm 0.06 \mathrm{ghi}$ & $0.66 \pm 0.01 \mathrm{~d}-\mathrm{g}$ & $83.33 \pm 2.08 \mathrm{a}$ & $0.40 \pm 0.00$ cde \\
\hline & Sour orange & $2.31 \pm 0.03 \mathrm{ij}$ & $0.63 \pm 0.06 \mathrm{i}-1$ & $0.41 \pm 0.04$ hij & $84.00 \pm 2.65 \mathrm{a}$ & $0.38 \pm 0.06 \mathrm{c}-\mathrm{f}$ \\
\hline & Keen sour orange & $2.24 \pm 0.06 \mathrm{ijk}$ & $0.63 \pm 0.06 \mathrm{i}-1$ & $0.49 \pm 0.04 \mathrm{ghi}$ & $82.33 \pm 3.06 \mathrm{a}$ & $0.34 \pm 0.03 \mathrm{c}-\mathrm{g}$ \\
\hline & Rough lemon & $2.71 \pm 0.25 \mathrm{fg}$ & $0.57 \pm 0.06 \mathrm{jkl}$ & $0.38 \pm 0.08 \mathrm{ij}$ & $84.00 \pm 1.00 \mathrm{a}$ & $0.29 \pm 0.04 \mathrm{fgh}$ \\
\hline & Brazilian sour orange & $2.70 \pm 0.06 \mathrm{~g}$ & $0.87 \pm 0.06 \mathrm{gh}$ & $0.69 \pm 0.08 c-g$ & $77.00 \pm 1.00 \mathrm{ab}$ & $0.51 \pm 0.06 \mathrm{a}$ \\
\hline & Sunki $\times$ bentake & $1.87 \pm 0.041$ & $0.47 \pm 0.061$ & $0.23 \pm 0.02 \mathrm{jk}$ & $85.67 \pm 4.16 \mathrm{a}$ & $0.18 \pm 0.01 \mathrm{ijk}$ \\
\hline & X639 & $2.10 \pm 0.06 \mathrm{k}$ & $0.57 \pm 0.06 \mathrm{jkl}$ & $0.36 \pm 0.04 \mathrm{ij}$ & $81.00 \pm 6.08 \mathrm{a}$ & $0.30 \pm 0.03 \mathrm{e}-\mathrm{h}$ \\
\hline & Kirrumakki nucellar & $2.10 \pm 0.04 \mathrm{k}$ & $0.53 \pm 0.06 \mathrm{kl}$ & $0.41 \pm 0.04$ hij & $82.33 \pm 2.31 \mathrm{a}$ & $0.51 \pm 0.01 \mathrm{ab}$ \\
\hline & Rangpur poona nucellar & $1.57 \pm 0.04 \mathrm{~m}$ & $0.43 \pm 0.061$ & $0.14 \pm 0.03 \mathrm{k}$ & $82.00 \pm 1.73 \mathrm{a}$ & $0.10 \pm 0.00 \mathrm{kl}$ \\
\hline & \multicolumn{6}{|l|}{$p$-value } \\
\hline & $p_{\text {Drought }}$ & $<0.0001$ & $<0.0001$ & $<0.0001$ & $<0.0001$ & $<0.0001$ \\
\hline & $p_{\text {Rootstock }}$ & $<0.0001$ & $<0.0001$ & $<0.0001$ & $=0.0261$ & $<0.0001$ \\
\hline & $p_{\text {Drought }} \times$ Rootstock & $<0.0001$ & $<0.0001$ & $<0.0001$ & $=0.0978$ & $<0.0001$ \\
\hline
\end{tabular}

Data presented are means \pm standard deviation (mean \pm SD) of three replicates. Different letters indicate statistically significant differences among treatments, while "ns" signifies no significant differences between them according to Tukey's honestly significant difference test $(p<0.05)$.

\subsection{Drought Stress Induced the Accumulation of Stress-Associated Biomarkers in Citrus Rootstocks}

Two major stress-associated biomarkers, including $\mathrm{H}_{2} \mathrm{O}_{2}$ and endogenous proline content, were assessed (Table 3$)$. Generally, both drought levels ( $\left.p_{\text {Drought }}<0.0001\right)$ and rootstocks $\left(p_{\text {Rootstock }}=0.0261\right)$ induced the $\mathrm{H}_{2} \mathrm{O}_{2}$ accumulation. Plants under drought 
stress conditions (moderate or severe) had significantly higher $\mathrm{H}_{2} \mathrm{O}_{2}$ levels, compared with non-stressed rootstocks. It is worth mentioning that all tested rootstocks had their highest $\mathrm{H}_{2} \mathrm{O}_{2}$ levels under severe drought conditions with no noticeable difference between them (Table 3 ). Similarly, endogenous proline content was significantly affected by waterdeficient $\left(p_{\text {Drought }}<0.0001\right)$ and citrus rootstock $\left(p_{\text {Rootstock }}<0.0001\right)$, and its levels were boosted with raising the severity of drought stress. Brazilian sour orange had the highest proline levels $\left(0.51 \pm 0.06 \mu \mathrm{mol} \mathrm{g}^{-1} \mathrm{FW}\right)$ under severe drought conditions. Nevertheless, there was no significant difference in proline content of different citrus rootstocks under regular irrigation conditions (Table 3).

3.5. Principal Component Analysis (PCA) and Two-Way Hierarchical Cluster Analysis (HCA) Revealed the Differences among Water Treatments and Citrus Rootstocks

To better understand the water deficiency and citrus rootstocks interactions, principal component analysis (PCA) and two-way hierarchical cluster analysis (HCA) were carried out (Figure 2). PCA-associated scatterplot revealed a clear separation among water deficit treatment (control, moderate drought, and severe drought), as well as all studied rootstocks with respect to PC1 (approximately $81.28 \%$ ) and PC2 (about 11.94\%) (Figure 2A). Moreover, the PCA-associated loading plot showed that while root length, root moisture content, shoot moisture content, shoot length, carotenoids, chlorophyll a, chlorophyll b, and water potential were positively correlated with non-stressed control plants, $\mathrm{H}_{2} \mathrm{O}_{2}$, and proline content were positively associated with water deficit treatments (Figure 2B).

In harmony with PCA findings, the HCA and its associated heatmap revealed the differences among water-deficient treatments (Figure 2C). For example, HCA-associated dendrogram among rootstocks revealed that all studied rootstocks were clustered separately into three distinct clusters. Cluster (a) included all non-stressed rootstocks, Cluster (b) included all moderate drought-stressed rootstocks except Rangpur Poona nucellar and Sunki $\times$ bentake which were clustered with severely stressed rootstocks within Cluster (c). Additionally, the HCA-associated dendrogram among investigated variables revealed that they were clustered into two separate clusters. Cluster ' $I$ ' included root length, root moisture content, shoot moisture content, shoot length, carotenoids, chlorophyll a, chlorophyll $\mathrm{b}$, and water potential which all were higher in regularly irrigated non-stressed control rootstocks. On the other hand, Cluster ' $\mathrm{II}$ ' included only $\mathrm{H}_{2} \mathrm{O}_{2}$ and proline contents which were higher in citrus rootstocks grown under severe deficient water stress (Figure 2C).

\subsection{Water Deficiency Alters the Anatomical Structure of Citrus Rootstocks}

To better understand the mechanism of drought resistance in citrus, the effect of different drought levels on the anatomical structure of stem and leaves of two highly tolerant (Brazilian sour orange and Gadha dahi) and two highly sensitive genotypes (Rangpur Poona nucellar and Sunki $\times$ bentake) from the first screening study was investigated. As we mentioned above, under drought stress conditions, the moisture content of both roots and shoots was reduced, resulting in yellow, curled, wilted leaves, and some other adverse wilt-associated symptoms (Figure 1). Briefly, under severe drought stress, the length of epidermal cells and vascular bundles was reduced, as well as the thickness of the xylem and cortical was thinner.

\subsubsection{Effect of Drought Stress on Stem Anatomy of Citrus Rootstocks}

Microscopic observation of stem cross-section showed that the highly tolerant genotypes (Brazilian sour orange and Gadha dahi) had some features such as water-filled cells, small cell gaps, tight and round cells under normal water conditions, as well as under drought stress conditions (Figure 3). 

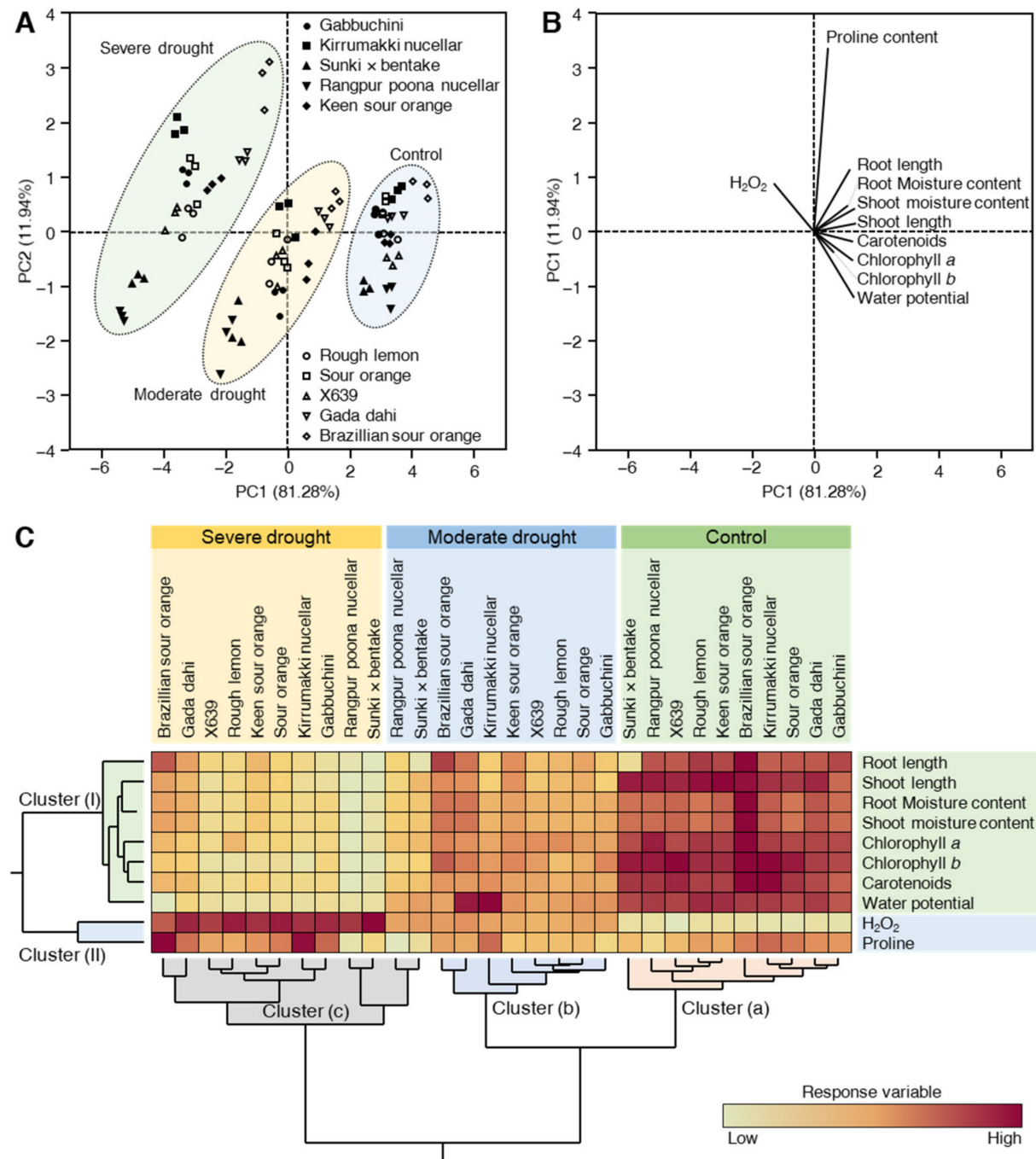

Figure 2. Principal component analysis (PCA) and two-way hierarchical cluster analysis (HCA) of individual morphological and physiological parameters were assessed in 10citrus rootstocks with distinct degrees of tolerance to drought stress under three water regimes. (A) PCA-associated scatterplots, (B) PCA-associated loading plots, and (C) two-way HCA. Variations in the dependent variables among studied treatments are visualized as a heat map. Rows correspond to dependent variables, whereas columns correspond to different treatments. Low numerical values are lightyellow-colored, while high numerical values are colored dark red (see the scale at the right bottom corner of the heat map).

However, both drought levels and rootstocks ( $p_{\text {Drought }}<0.0001$ and $p_{\text {Rootstock }}=0.0002$, respectively) significantly affected the length of the epidermal cell and vascular bundle (Figure 4A,B, respectively), xylem thickness (Figure 4C), area of metaxylem cell (Figure 4D), phloem cell (Figure 4E), pith cell (Figure 4F), pith thickness (Figure 4G), and cortical cell (Figure $4 \mathrm{H}$ ), and cortical thickness (Figure $4 \mathrm{I}$ ). Under all water conditions, the anatomical structures of the highly tolerant genotypes Brazilian sour orange and Gadha dahi performed better than two highly sensitive genotypes Rangpur Poona nucellar and Sunki $\times$ bentake. For instance, under regular irrigation, both Brazilian sour orange and Gadha dahi rootstocks had the highest epidermal cell length ( $p_{\text {Drought }} \times$ Rootstock $\left.=0.0255\right)$, vascular bundle length $\left(p_{\text {Drought }} \times\right.$ Rootstock $\left.<0.0001\right)$, xylem thickness $\left(p_{\text {Drought }} \times\right.$ Rootstock $\left.=0.488\right)$, metaxylem cell area $\left(p_{\text {Drought }} \times\right.$ Rootstock $\left.=0.0140\right)$, phloem cell area $\left(p_{\text {Drought }} \times\right.$ Rootstock $\left.=0.0346\right)$, pith cell area $\left(p_{\text {Drought }} \times\right.$ Rootstock $\left.=0.0316\right)$, pith thickness area $\left(p_{\text {Drought }} \times\right.$ Rootstock $\left.=0.0112\right)$, cortical cell area $\left(p_{\text {Drought }} \times\right.$ Rootstock $\left.=0.0180\right)$, and cortical thickness $\left(p_{\text {Drought }} \times\right.$ Rootstock $\left.=0.0319\right)$ with no significant differences between them. Although, both moderate and severe drought 
stresses significantly reduced most, if not all, of these anatomical attributes, both highly tolerant genotypes did not show noticeable changes under drought stress. Nevertheless, the mesophyll cells of drought-sensitive Rangpur Poona nucellar and Sunki $\times$ bentake genotypes were slightly deformed and had shorter epidermal cell and vascular bundle length, as well as narrower metaxylem, phloem, pith, and cortical area (Figure 4).

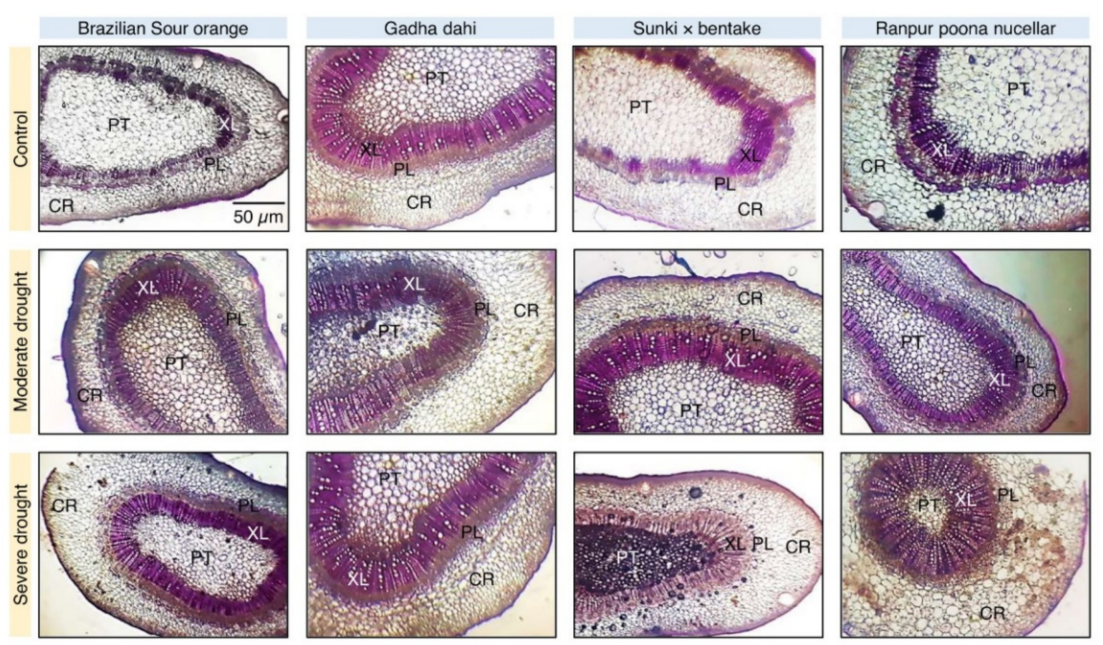

Figure 3. Stem transverse section of two highly tolerant (Brazilian sour orange and Gadha dahi) and two highly sensitive genotypes (Rangpur Poona nucellar and Sunki $\times$ bentake). PT: pith; CR: cortex; XL: xylem; PL: phloem.

3.6.2. PCA and Two-Way HCA Divulged the Variations in Stem Anatomy of Different Citrus Rootstocks

Briefly, the PCA-associated scatter plot showed a clear separation among water deficit treatment (control, moderate drought, and severe drought), as well as all studied rootstocks with respect to PC1 (approximately 87.29\%) and PC2 (about 8.41\%) (Figure 5A). It is worth mentioning that the drought-tolerant rootstocks Brazilian sour orange and Gadha dahi were grouped close to each other and separately from the two sensitive genotypes Rangpur Poona nucellar and Sunki $\times$ bentake under normal irrigation and moderate drought, but not severe drought conditions. Furthermore, the PCA-associated loading plot showed that all studied stem anatomical features were positively associated with normal water application (Figure 5B). In agreement with PCA results, the HCA and its associated heatmap uncovered the differences among different rootstocks under water-deficient treatments (Figure 5C). For example, HCA-associated dendrogram among rootstocks revealed that all studied rootstocks separated into two distinct clusters. Cluster (a) included all non-stressed rootstocks and the highly tolerant (Brazilian sour orange and Gadha dahi), grown under moderate drought, whereas cluster (b) included all severely stressed rootstocks and the two highly sensitive genotypes (Rangpur Poona nucellar and Sunki $\times$ bentake) that were moderately stressed with drought (Figure 5C). 

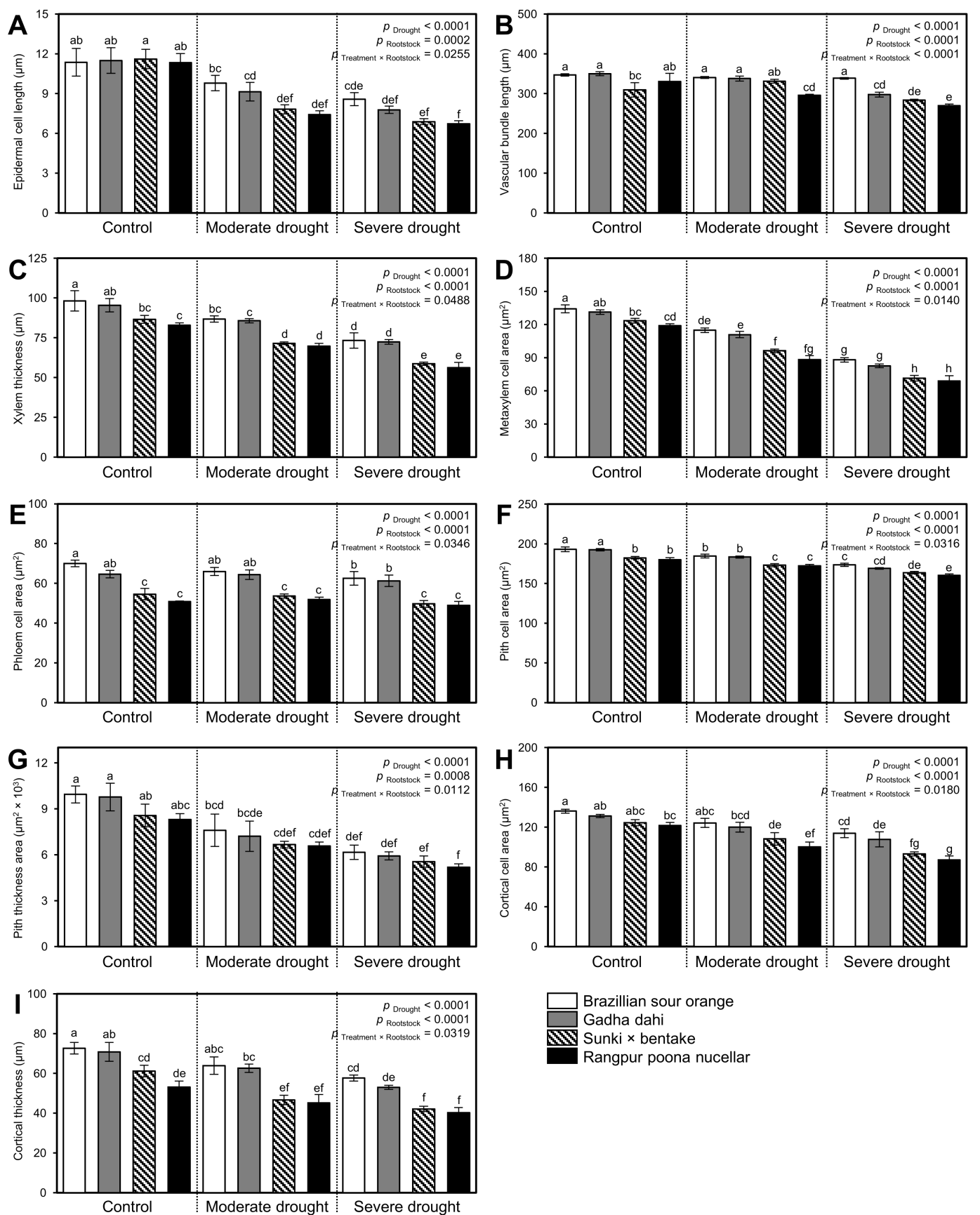

Figure 4. Effect of different water regimes on the stem anatomical features of two highly tolerant (Brazilian sour orange and Gadha dahi) and two highly sensitive genotypes (Rangpur Poona nucellar and Sunki $\times$ bentake). (A) Epidermal cell length $(\mu \mathrm{m}),(\mathbf{B})$ Vascular bundle length $(\mu \mathrm{m}),(\mathbf{C})$ Xylem thickness $(\mu \mathrm{m}),(\mathbf{D})$ Metaxylem cell area $\left(\mu \mathrm{m}^{2}\right),($ E) Phloem cell area $\left(\mu \mathrm{m}^{2}\right),(\mathbf{F})$ Pith cell area $\left(\mu \mathrm{m}^{2}\right),(\mathbf{G})$ Pith thickness area $\left(\mu \mathrm{m}^{2} \times 10^{3}\right),(\mathbf{H})$ Cortical cell area $\left(\mu \mathrm{m}^{2}\right)$, and (I) Cortical thickness $(\mu \mathrm{m})$. Data presented are means \pm standard deviation (mean $\pm \mathrm{SD}$ ) of three biological replicates. Different letters indicate statistically significant differences among treatments, while "ns" signifies no significant differences between them according to Tukey's honestly significant difference test $(p<0.05)$. 

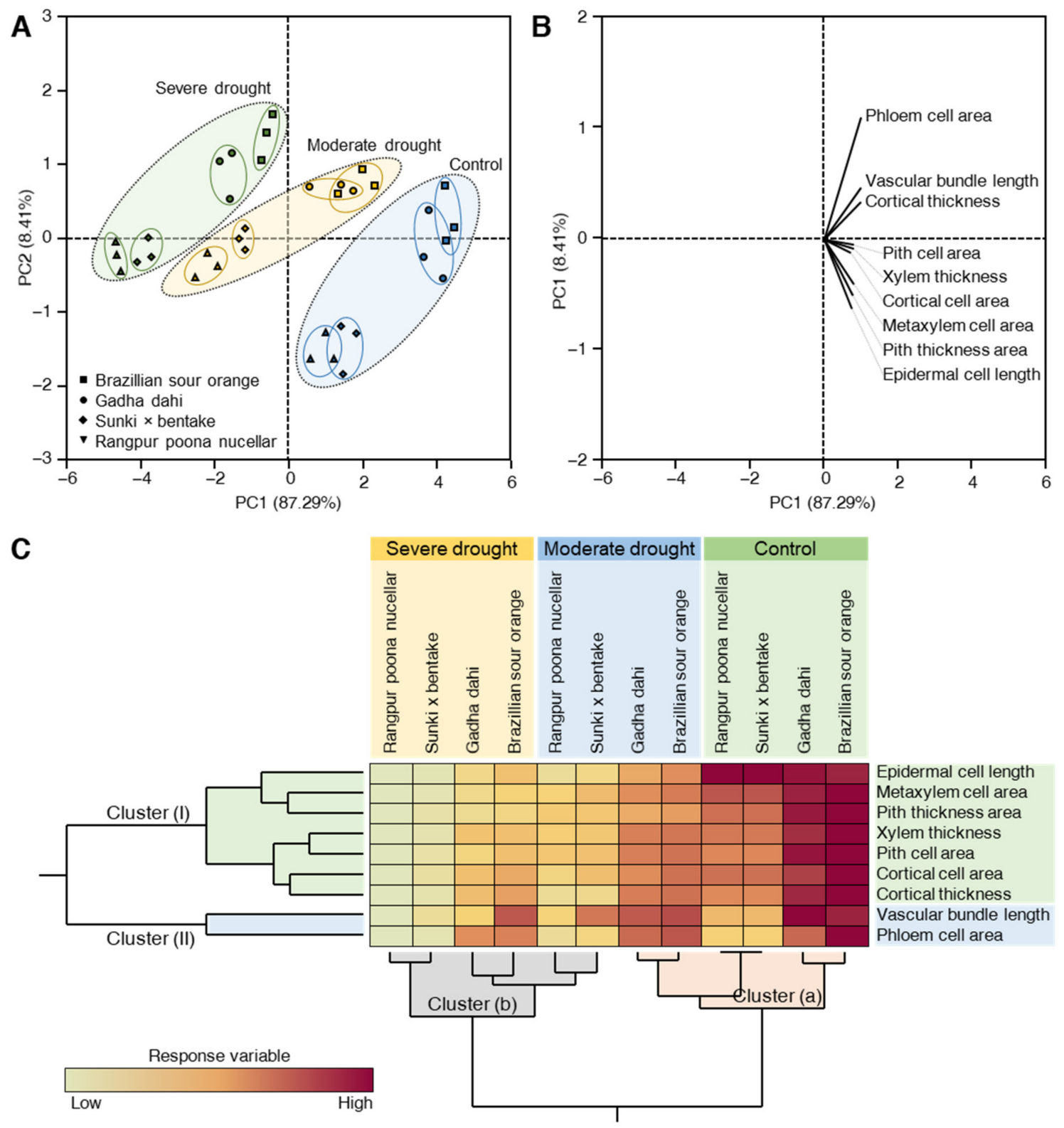

Figure 5. Principal component analysis (PCA) and two-way hierarchical cluster analysis (HCA) of individual stem anatomical features of two highly tolerant (Brazilian sour orange and Gadha dahi) and two highly sensitive genotypes (Rangpur Poona nucellar and Sunki $\times$ bentake) under three water regimes. (A) PCA-associated scatter plots, (B) PCAassociated loading plots, and (C) two-way HCA. Variations in the dependent variables among studied treatments are visualized as a heat map. Rows correspond to dependent variables, whereas columns correspond to different treatments. Low numerical values are light-yellow-colored, while high numerical values are colored dark red (see the scale at the right bottom corner of the heat map).

\subsubsection{Effect of Drought Stress on Leaf Tissue Structure of Citrus Rootstocks}

Microscopic observation of citrus leaves cross-section showed that it had an asymmetric heterogeneous structure that was characterized by two unequal palisade parenchyma (Figure 6). 


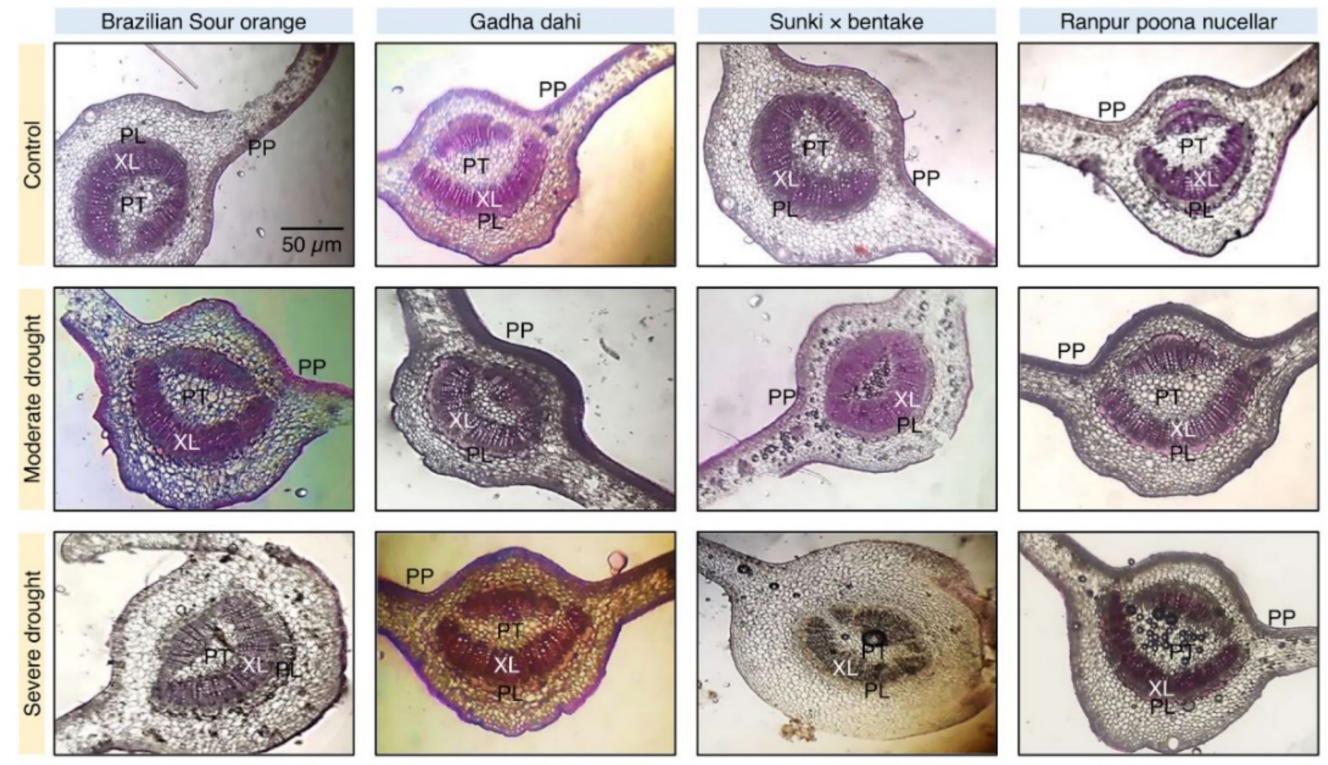

Figure 6. Leaf transverse section of two highly tolerant (Brazilian sour orange and Gadha dahi) and two highly sensitive genotypes (Rangpur Poona nucellar and Sunki $\times$ bentake). PT: pith; CR: cortex; XL: xylem; PL: phloem; PP: palisade parenchyma.

Like stem anatomy, citrus leaf anatomical attributes were significantly altered by both drought levels and rootstocks. However, the anatomical changes in leaf tissue structure were less significant in highly tolerant rootstocks (Brazilian sour orange and Gadha dahi) than sensitive genotypes (Rangpur Poona nucellar and Sunki $\times$ bentake). Interestingly, differences in all studied anatomical features of citrus leaves under different water regimes were cultivar-dependent. These features included epidermal cell length $\left(p_{\text {Drought }} \times\right.$ Rootstock $=0.0315 ;$ Figure 7A), vascular bundle length $\left(p_{\text {Drought }} \times\right.$ Rootstock $=0.0009 ;$ Figure $\left.7 \mathrm{~B}\right)$, xylem thickness $\left(p_{\text {Drought }} \times\right.$ Rootstock $=0.0251 ;$ Figure $\left.7 \mathrm{C}\right)$, metaxylem cell area $\left(p_{\text {Drought }} \times\right.$ Rootstock $=0.0349 ;$ Figure 7D), phloem cell area $\left(p_{\text {Drought }} \times\right.$ Rootstock $=0.0197$; Figure 7E), pith cell area $\left(p_{\text {Drought }} \times\right.$ Rootstock $=0.0163 ;$ Figure $\left.7 \mathrm{~F}\right)$, pith thickness area $\left(p_{\text {Drought }} \times\right.$ Rootstock $=0.0335$; Figure $7 \mathrm{G})$, cortical cell area $\left(p_{\text {Drought }} \times\right.$ Rootstock $=0.0250$; Figure $\left.7 \mathrm{H}\right)$, and cortical thickness $\left(p_{\text {Drought }} \times\right.$ Rootstock $=0.0357 ;$ Figure $\left.7 \mathrm{I}\right)$. Under all tested water regimes, highly tolerant rootstocks Brazilian sour orange and Gadha dahi had thicker epidermal and vascular bundle, as well as wider pith and cortical areas compared with sensitive genotypes (Rangpur Poona nucellar and Sunki $\times$ bentake). Additionally, severe drought stress significantly reduced the thickness of all leaf tissues, particularly in sensitive genotypes (Figure 7).

3.6.4. PCA and Two-Way HCA Revealed the Differences in Leaf Tissue Structure of Different Citrus Rootstocks

In brief, the PCA-associated scatter plot showed a clear separation among water deficit treatment (control, moderate drought, and severe drought), as well as all studied rootstocks with respect to PC1 (approximately 93.24\%) and PC2 (about 3.95\%) (Figure 8A). It is worth mentioning that the drought-tolerant rootstocks Brazilian sour orange and Gadha dahi were grouped close to each other and separately from the two sensitive genotypes Rangpur Poona nucellar and Sunki $\times$ bentake under all investigated water regimes. Moreover, the PCA-associated loading plot showed that all studied anatomical features of citrus leaves were positively associated with normal water application (Figure 8B). Like PCA, the HCA and its associated heatmap revealed the differences among different rootstocks under water-deficient treatments (Figure $8 \mathrm{C}$ ). For example, HCA-associated dendrogram among rootstocks revealed that all studied rootstocks separated into two distinct clusters. Cluster (a) included all regularly irrigated genotypes and the two highly tolerant rootstocks (Brazilian sour orange and Gadha dahi) that were grown under moderate drought. On the other hand, cluster (b) included all severely stressed genotypes and the two sensitive 
rootstocks (Rangpur Poona nucellar and Sunki $\times$ bentake) that were moderately stressed with drought (Figure 8C).
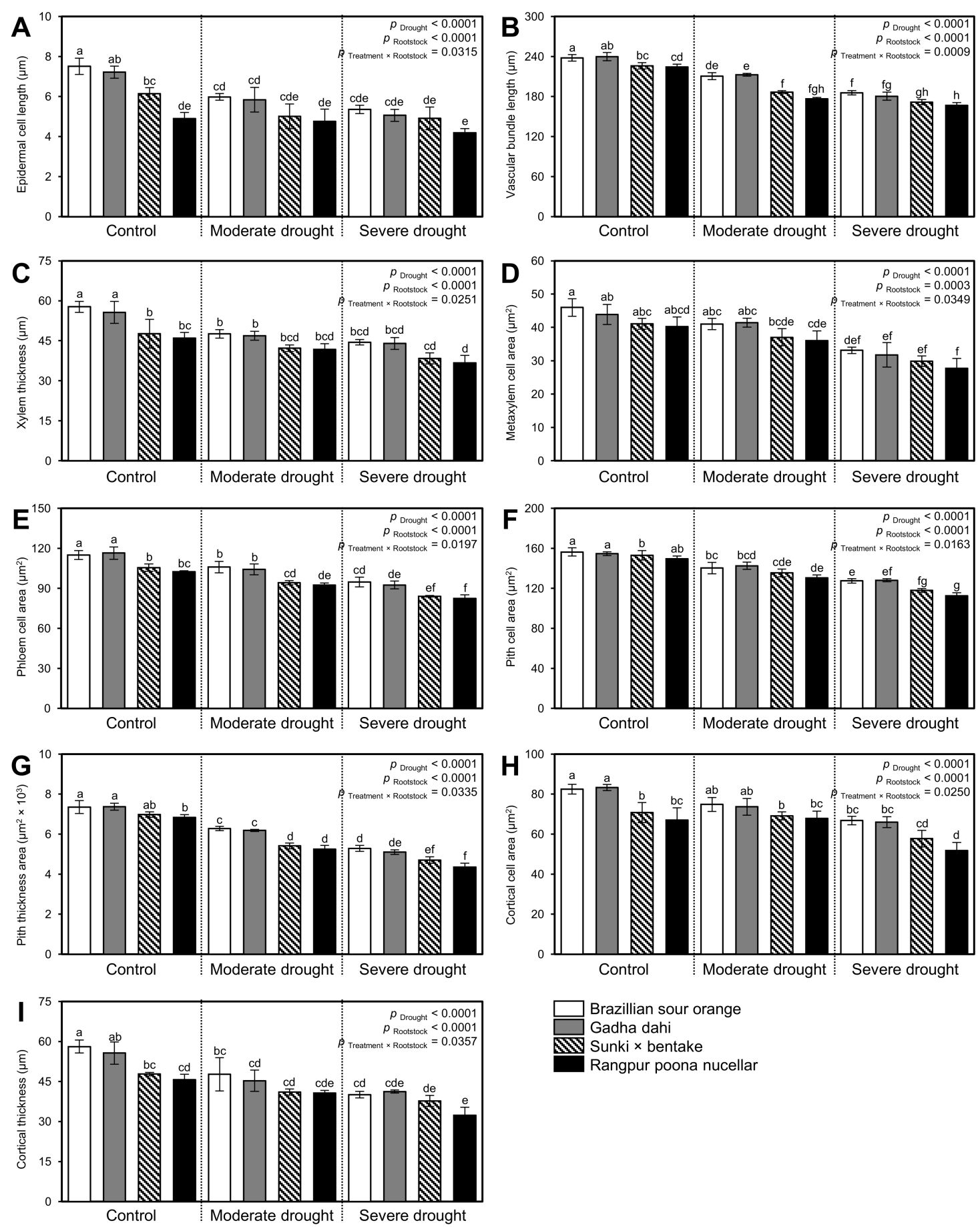

Figure 7. Effect of different water regimes on the leaf anatomical features of different two highly tolerant (Brazilian sour orange and Gadha dahi) and two highly sensitive genotypes (Rangpur Poona nucellar and Sunki $\times$ bentake). (A) Epidermal cell length $(\mu \mathrm{m}),(\mathbf{B})$ Vascular bundle length $(\mu \mathrm{m}),(\mathbf{C})$ Xylem thickness $(\mu \mathrm{m}),(\mathbf{D})$ Metaxylem cell area $\left(\mu \mathrm{m}^{2}\right),($ E) Phloem cell area $\left(\mu \mathrm{m}^{2}\right),(\mathbf{F})$ Pith cell area $\left(\mu \mathrm{m}^{2}\right),(\mathbf{G})$ Pith thickness area $\left(\mu \mathrm{m}^{2} \times 10^{3}\right),(\mathbf{H})$ Cortical cell area $\left(\mu \mathrm{m}^{2}\right)$, and (I) Cortical thickness $(\mu \mathrm{m})$. Data presented are means \pm standard deviation (mean $\pm \mathrm{SD}$ ) of three biological replicates. Different letters indicate statistically significant differences among treatments, while "ns" signifies no significant differences between them according to Tukey's honestly significant difference test $(p<0.05)$. 

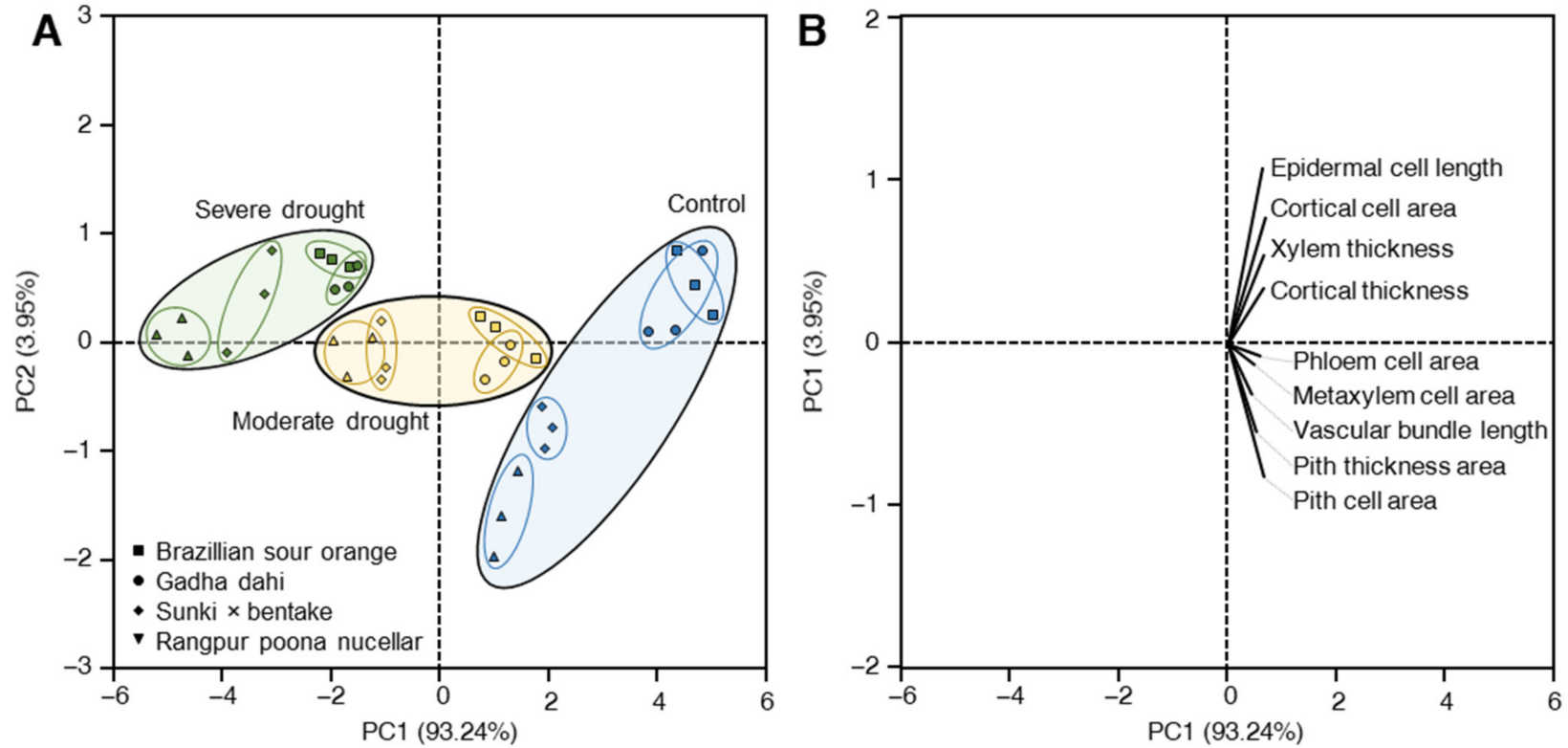

C
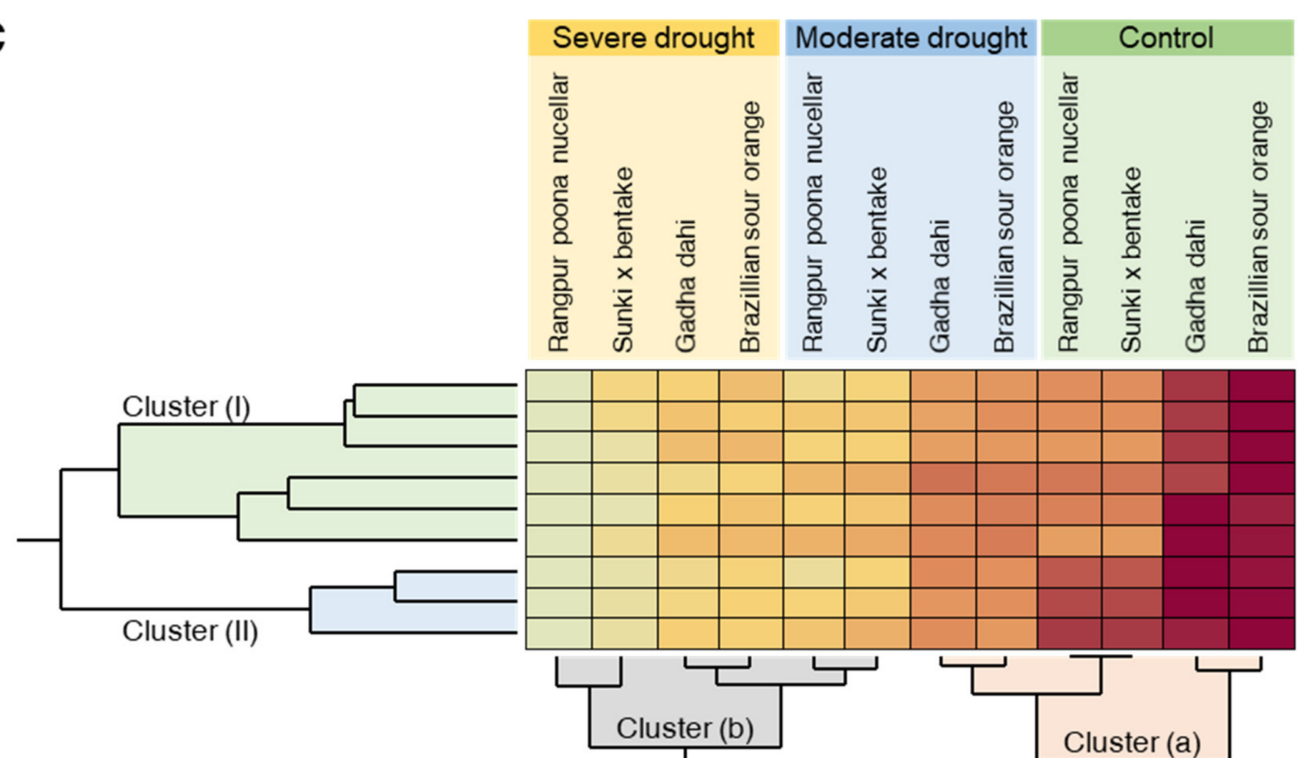

Epidermal cell length Cortical thickness Xylem thickness Metaxylem cell area Phloem cell area Cortical cell area Vascular bundle length Pith thickness area Low Response variable

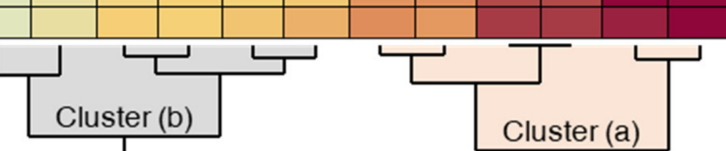

Pith cell area

Figure 8. Principal component analysis (PCA) and two-way hierarchical cluster analysis (HCA) of individual leaf anatomical features of two highly tolerant (Brazilian sour orange and Gadha dahi) and two highly sensitive genotypes (Rangpur Poona nucellar and Sunki $\times$ bentake) under three water regimes. (A) PCA-associated scatter plots, (B) PCA-associated loading plots, and (C) two-way HCA. Variations in the dependent variables among studied treatments are visualized as a heat map. Rows correspond to dependent variables, whereas columns correspond to different treatments. Low numerical values are light-yellow-colored, while high numerical values are colored dark red (see the scale at the right bottom corner of the heat map).

\section{Discussion}

Water deficit conditions are a major environmental factor, which frequently limits the growth and productivity of important crop species [17]. Restriction of water supply can severely limit plant growth, development, and production [18,19]. Choice of rootstock is among the most important decisions a grower makes, and implications for yield and 
quality are enormous. Rootstock in citrus trees influences the morphological, biochemical, physiological, and genetic characteristics of grafted scion cultivars through the rootstock scion interaction pathway [51]. Citrus rootstocks with better drought tolerance ability can greatly reduce production losses [52]. In this study, plant material consisted of 10 genetically diverse citrus rootstocks belonging to different citrus categories, i.e., oranges, pummelo, lemon, lime, their hybrids, and originating from diverse localities. The leaf shape and size of these rootstocks also varied. These rootstocks are reported to have different tolerance towards some biotic and abiotic stresses. The drought tolerance of these rootstocks was studied in this investigation. Leaf water potential in plants is directly related to water availability [53]. Leaf water potential indicates the whole plant water status, and maintenance of high leaf water potential is found to be associated with dehydration avoidance mechanisms. Our results demonstrated the decrease in leaf water potential as drought conditions become severe compared with control. The maintenance of water potential in leaves is a direct indicator of a plant dehydration avoidance mechanism, as genotypes with better water potential at stress conditions are regarded as drought tolerant $[23,54,55]$. In our studies, Brazilian sour orange showed drought tolerance by performing best at severe drought and Rangpur Poona nucellar at moderate drought. For insensitive genotypes, the decrease in leaf water potential indicated the mechanical injury of leaf chloroplasts caused by stress conditions which result in reduced transpiration rate and oxidative stress [16].

Citrus rootstocks with high chlorophyll $a$ and $b$, and carotenoid contents against the stresses, especially the water stress, are regarded as tolerant rootstocks [56]. The normal functioning of photosynthetic machinery is affected by drought stress, the degradation, and photo-oxidation of chlorophyll caused by transpirational imbalance at water stress hamper the plant's ability to harvest light reducing total photosynthetic output [21,57]. Results showed that photosynthetic pigments chlorophyll $a$, chlorophyll $b$, and carotenoids reduced significantly at elevated stress conditions and overall genotype Brazilian sour orange and Gada dahi had the highest chlorophyll contents at drought conditions highlighting their ability to tolerate drought stress. Plants with dark green leaves (chlorophyll) under drought stress are considered tolerant. Visual assessments indicated the Brazilian sour orange as a tolerant rootstock without changes in leaf green color and leaf necrosis; while, Savage citrange emerged as the most sensitive, with maximum plant death and leaf shedding during stress treatments. The compromised photosynthetic machinery reduces carbohydrate transport and as a result plant growth is also reduced. The ability of plants to maintain growth under limited water supply reveals their tolerance ability $[43,58]$. Results showed that among citrus rootstocks, Brazilian sour orange and Gada dahi at water stress conditions maintained steady root and shoot growth. The root and shoot moisture content of these rootstocks were also high at stress conditions showing their tolerant nature. While, Rangpur Poona nucellar had the lowest shoot and root growth and moisture content emerged as the most sensitive.

Metabolic imbalances triggered by drought stress cause oxidative stress and as a result ROS are produced and accumulated [59]. The increased oxidation greatly reduces metabolic activities and the normal functioning of cell organelles. To combat oxidative stress, plants also have a built-in antioxidant defense mechanism. Proline is an osmoprotectant that is trigged as a result of ROS production in the cell, its production and accumulation work as ROS scavenging, redox balance, and reduce cell damage which normalizes the functionality of plant cells [60]. In drought-tolerant genotypes, the ROS production is reduced, and proline concentration is increased with increasing severity of stress. The results show that Brazilian sour orange has minimal ROS production and the highest proline concentration at severe drought stress.

In citrus under drought stress, leaves are observed to be shorter with thick epidermal cells which facilitates reducing the transpirational rate and oxidative stress [61,62]. Modifications in vascular anatomy are important for plant acclimation potential. Vascular bundles present in mid rid of leaves serve as a source to distribute nutrients and water. At 
stress conditions with reduced leaf size, the reduction in the vascular bundle is an indicator of plants' abilities to modify their anatomy under stress. In the vascular bundle, the xylem acts as a source of water transport. The plants with greater xylem vessel diameter are unable to survive harsh environmental conditions [63-65]. At the onset of drought, stress transpiration, water uptake from roots, and stem hydraulic capacitance begin to decline. That reduces growth, the vascular bundles in the stem are observed to be reduced along with pith cell area and cortical thickness [66,67]. In this study, the results showed that with increasing drought intensity the anatomy of both sensitive and tolerant genotypes was modified, interestingly the two tolerant genotypes Brazilian sour orange and Gada dahi had greater values for all the leaf and stem anatomy parameters at severe drought stress than sensitive genotypes. This could be because of the continuous adaptability of tolerant genotypes which enabled them to maintain growth and function as the amount of water became limited, in response to sensitive genotypes in which the response could have been triggered at very later stages which abruptly affect their growth. These modifications in tolerant genotypes enabled them to maintain steady nutrient transport while reducing the risk of embolisms, increasing water-flow resistance, and constant transport of nutrients across [64].

\section{Conclusions}

Drought stress adversely affected plant water status, photosynthetic machinery, biochemical balance, and anatomical structure of all the citrus rootstocks studied. The intensifying drought reduced leaf water potential, and compromised the photosynthetic apparatus by damaging photosynthetic pigments (chlorophyll " $a$ ", " $b$ ", and carotenoid) apparent from lighter green color. Oxidative stress caused by ROS production which triggered production of proline. Alteration in anatomical structures of leaf and stem were observed. Citrus rootstocks Brazilian sour orange and Gada dahi performed best under drought stress, mitigated damage at molecular biochemical and anatomical levels, while rootstocks Sunki $\times$ bentake and Rangpur Poona nucellar were the most sensitive rootstocks.

Author Contributions: Conceptualization, M.J.J., S.A.N., M.H. and Y.N., methodology, W.S., M.J.J., Y.S.A.M. and M.H.; software, W.S., S.A.N., Y.N. and S.I.; formal analysis, Y.S.A.M., W.S., S.-u.-R. and Y.N.; resources, M.J.J., M.H., S.A.N. and Y.S.A.M.; data curation, S.-u.-R., S.B., S.I. and W.S.; writing—original draft preparation, W.S., S.I., S.-u.-R. and Y.N.; writing—review and editing, M.J.J, Y.S.A.M., S.A.N., Y.N. and M.H.; supervision, M.J.J. All authors have read and agreed to the published version of the manuscript.

Funding: We would like to acknowledge the Higher Education Commission, Islamabad and Endowment Fund Secretariat, University of Agriculture, Faisalabad, Pakistan for providing funding. The authors extend their appreciation to the Deanship of Scientific Research at King Khalid University for funding this work through the Program of Research Groups under grant number (RGP 2/165/42).

Institutional Review Board Statement: Not applicable.

Informed Consent Statement: Not applicable.

Data Availability Statement: The data that supports the findings of this study are contained within the article and available from the corresponding author upon reasonable request.

Acknowledgments: Y.N. would like to extend his appreciation to the Graduate Student and Research Affairs Sector of Tanta University, Egypt. We also thank all staff members of our laboratories for their helpful discussions and comments. The authors extend their appreciation to the Deanship of Scientific Research at King Khalid University for funding this work through the Program of Research Groups under grant number (RGP 2/165/42).

Conflicts of Interest: The authors declare that there is no conflict of interest, and they have no known competing financial interests or personal relationships that could have appeared to influence the work reported in this paper. 


\section{References}

1. Mehdi, M.; Ashfaq, M.; Hassan, S.; Abid, M. Effect of marketing channel choice on the profitability of citrus farmers: Evidence form Punjab-Pakistan. Pak. J. Agric. Sci. 2019, 56, 1003-1011.

2. Talat, H.; Shafqat, W.; Qureshi, M.A.; Sharif, N.; Raza, M.K.; Din, S.; Ikram, S.; Jaskani, M.J. Effect of gibberellic acid on fruit quality of Kinnow mandarin. J. Glob. Innov. Agric. Soc. Sci. 2020, 8, 59-63. [CrossRef]

3. Silva, S.F.; Miranda, M.T.; Costa, V.E.; Machado, E.C.; Ribeiro, R.V. Sink strength of citrus rootstocks under water deficit. Tree Physiol. 2021, 41, 1372-1383. [CrossRef] [PubMed]

4. Qureshi, M.A.; Jaskani, M.J.; Khan, A.S.; Ahmad, R. Influence of endogenous plant hormones on physiological and growth attributes of Kinnow mandarin grafted on nine rootstocks. J. Plant Growth Regul. 2021, 1-11. [CrossRef]

5. Qureshi, M.A.; Jaskani, M.J.; Khan, A.S.; Haider, M.S.; Shafqat, W.; Asif, M.; Mehmood, A. Influence of different rootstocks on physico-chemical quality attributes of Kinnow mandarin. Pak. J. Agric. Sci. 2021, 58, 929-935.

6. Khan, K.; Ikram, S.; Ashfaq, M.; Jaskani, M.J.; Shafqat, W. Citrus rootstock characterization against citrus canker and evaluation of antibiotics effect against Xanthomonas axonopodis pv. Citri. J. Innov. Agric. 2021, 8, 1-8.

7. Shafqat, W.; Tahir, T.; Khurshid, T.; Ur-Rahman, H.; Saqib, M.; Jaskani, M.J. Effect of rootstock types on leaf nutrient composition in three commercial citrus scion cultivars of Pakistan under the ASLP Citrus Project. In Proceedings of the XXIX International Horticultural Congress on Horticulture: Sustaining Lives, Livelihoods and Landscapes (IHC2014): 1128, Brisbane, Australia, 25 November 2016.

8. Bowman, K.D.; Joubert, J. Citrus rootstocks. In The Genus Citrus; Woodhead Publishing Books-Elsevier: Sawston, UK, 2020; pp. 105-127.

9. Morianou, G.; Ziogas, V.; Kourgialas, N.N.; Karatzas, G.P. Effect of irrigation practices upon yield and fruit quality of four grapefruit (Citrus paradisi Mac.) cultivars. Water Supply 2021, 21, 2735-2747. [CrossRef]

10. Koshita, Y.; Takahara, T. Effect of water stress on flower-bud formation and plant hormone content of satsuma mandarin (Citrus unshiu Marc.). Sci. Hortic. 2004, 99, 301-307. [CrossRef]

11. Syvertsen, J.; Hanlon, E.A. Citrus tree stresses: Effects on growth and yield. EDIS 2008, 2008, 1-6.

12. Zaman, L.; Shafqat, W.; Qureshi, A.; Sharif, N.; Raza, K.; ud Din, S.; Ikram, S.; Jaskani, M.J. Effect of foliar spray of zinc sulphate and calcium carbonate on fruit quality of Kinnow mandarin (Citrus reticulata Blanco). J. Glob. Innov. Agric. Soc. Sci. 2019, 7, 157-161. [CrossRef]

13. Bray, E.A.; Bailey-Serres, J.; Weretilnyk, E. Responses to abiotic stress. In Biochemistry E Molecular Biology of Plants; Gruissem, W., Jones, R., Eds.; American Society of Plant Physiologists: Rockville, MD, USA, 2000; pp. 1158-1203.

14. Bates, B.; Kundzewicz, Z.; Wu, S. Climate Change and Water; Intergovernmental Panel on Climate Change Secretariat: Geneva, Switzerland, 2008; ISBN 9291691232.

15. Ramírez, D.A.; Rolando, J.L.; Yactayo, W.; Monneveux, P.; Mares, V.; Quiroz, R. Improving potato drought tolerance through the induction of long-term water stress memory. Plant Sci. 2015, 238, 26-32. [CrossRef] [PubMed]

16. Zandalinas, S.I.; Rivero, R.M.; Martínez, V.; Gómez-Cadenas, A.; Arbona, V. Tolerance of citrus plants to the combination of high temperatures and drought is associated to the increase in transpiration modulated by a reduction in abscisic acid levels. $B M C$ Plant Biol. 2016, 16, 105. [CrossRef] [PubMed]

17. Barnabás, B.; Jäger, K.; Fehér, A. The effect of drought and heat stress on reproductive processes in cereals. Plant Cell Environ. 2008, 31, 11-38. [CrossRef] [PubMed]

18. Chen, Y.; Müller, F.; Rieu, I.; Winter, P. Epigenetic events in plant male germ cell heat stress responses. Plant Reprod. 2016, 29, 21-29. [CrossRef] [PubMed]

19. Li, X.; Liu, F. Drought stress memory and drought stress tolerance in plants: Biochemical and molecular basis. In Drought Stress Tolerance in Plants; Springer: New York, NY, USA, 2016; Volume 1, pp. 17-44.

20. Kinoshita, T.; Seki, M. Epigenetic memory for stress response and adaptation in plants. Plant Cell Physiol. 2014, 55, 1859-1863. [CrossRef]

21. Shafqat, W.; Jaskani, M.; Maqbool, R.; Sattar Khan, A. Evaluation of citrus rootstocks against drought, heat and their combined stress based on growth and photosynthetic pigments fingerprinting of Jamun (Syzygium cumini) genetic resources of Punjab view project. Int. J. Agri. Biol. 2019, 22, 1001-1009.

22. Chaves, M.M.; Flexas, J.; Pinheiro, C. Photosynthesis under drought and salt stress: Regulation mechanisms from whole plant to cell. Ann. Bot. 2009, 103, 551-560. [CrossRef]

23. Xiong, B.; Wang, Y.; Zhang, Y.; Ma, M.; Gao, Y.; Zhou, Z.; Wang, B.; Wang, T.; Lv, X.; Wang, X. Alleviation of drought stress and the physiological mechanisms in Citrus cultivar (Huangguogan) treated with methyl jasmonate. Biosci. Biotechnol. Biochem. 2020, 84, 1958-1965. [CrossRef]

24. Liu, S.; Yang, R. Regulations of reactive oxygen species in plants abiotic stress: An integrated overview. In Plant Life under Changing Environment: Responses and Management; Tripathi, D.K., Singh, V.P., Chauhan, D.K., Sharma, S., Prasad, S.M., Dubey, N.K., Ramawat, N., Eds.; Academic Press-Elsevier: Cambridge, MA, USA, 2020; pp. 323-353.

25. Noctor, G.; Veljovic-Jovanovic, S.; Driscoll, S.; Novitskaya, L.; Foyer, C.H. Drought and oxidative load in the leaves of C3 plants: A predominant role for photorespiration? Ann. Bot. 2002, 89, 841-850. [CrossRef] [PubMed] 
26. Guo, Y.Y.; Yu, H.Y.; Yang, M.M.; Kong, D.S.; Zhang, Y.J. Effect of drought stress on lipid peroxidation, osmotic adjustment and antioxidant enzyme activity of leaves and roots of Lycium ruthenicum Murr. seedling. Russ. J. Plant Physiol. 2018, 65, 244-250. [CrossRef]

27. Baker, N.R.; Rosenqvist, E. Applications of chlorophyll fluorescence can improve crop production strategies: An examination of future possibilities. J. Exp. Bot. 2004, 55, 1607-1621. [CrossRef] [PubMed]

28. de Matos Nunes, J.; Bertodo, L.O.O.; Da Rosa, L.M.G.; Von Poser, G.L.; Rech, S.B. Stress induction of valuable secondary metabolites in Hypericum polyanthemum acclimatized plants. S. Afr. J. Bot. 2014, 94, 182-189. [CrossRef]

29. Zaefyzadeh, M.; Quliyev, R.A.; Babayeva, S.M.; Abbasov, M.A. The effect of the interaction between genotypes and drought stress on the superoxide dismutase and chlorophyll content in durum wheat landraces. Turk. J. Biol. 2009, 33, 1-7.

30. Osmolovskaya, N.; Shumilina, J.; Kim, A.; Didio, A.; Grishina, T.; Bilova, T.; Keltsieva, O.A.; Zhukov, V.; Tikhonovich, I.; Tarakhovskaya, E. Methodology of drought stress research: Experimental setup and physiological characterization. Int. J. Mol. Sci. 2018, 19, 4089. [CrossRef]

31. Nyachiro, J.M.; Briggs, K.G.; Hoddinott, J.; Johnson-Flanagan, A.M. Chlorophyll content, chlorophyll fluorescence and water deficit in spring wheat. Cereal Res. Commun. 2001, 29, 135-142. [CrossRef]

32. Kyparissis, A.; Grammatikopoulos, G.; Manetas, Y. Leaf morphological and physiological adjustments to the spectrally selective shade imposed by anthocyanins in Prunus cerasifera. Tree Physiol. 2007, 27, 849-857. [CrossRef] [PubMed]

33. Liang, X.; Zhang, L.; Natarajan, S.K.; Becker, D.F. Proline mechanisms of stress survival. Antioxid. Redox Signal. 2013, 19, 998-1011. [CrossRef] [PubMed]

34. Verbruggen, N.; Hermans, C. Proline accumulation in plants: A review. Amino Acids 2008, 35, 753-759. [CrossRef]

35. Matsuda, K.; Rayan, A. Anatomy: A key factor regulating plant tissue response to water stress. In Environment Injury to Plants; Kafternan, F., Ed.; Academic Press-Elsevier: Cambridge, MA, USA, 1990; p. 290.

36. Olmos, E.; Sánchez-Blanco, M.J.; Ferrández, T.; Alarcon, J.J. Subcellular effects of drought stress in Rosmarinus officinalis. Plant Biol. 2007, 9, 77-84. [CrossRef]

37. Tombesi, S.; Johnson, R.S.; Day, K.R.; DeJong, T.M. Relationships between xylem vessel characteristics, calculated axial hydraulic conductance and size-controlling capacity of peach rootstocks. Ann. Bot. 2009, 105, 327-331. [CrossRef] [PubMed]

38. Carlquist, S. Comparative Wood Anatomy: Systematic, Ecological, and Evolutionary Aspects of Dicotyledon Wood; Springer Science \& Business Media: Berlin/Heidelberg, Germany, 2013; ISBN 3662217147.

39. Pitman, W.D.; Holt, E.C.; Conrad, B.E.; Bashaw, E.C. Histological differences in moisture-stressed and nonstressed Kleingrass forage 1. Crop. Sci. 1983, 23, 793-795. [CrossRef]

40. Guerfel, M.; Baccouri, O.; Boujnah, D.; Chaibi, W.; Zarrouk, M. Impacts of water stress on gas exchange, water relations, chlorophyll content and leaf structure in the two main Tunisian olive (Olea europaea L.) cultivars. Sci. Hortic. 2009, 119, 257-263. [CrossRef]

41. Child, R.D.; Summers, J.E.; Babij, J.; Farrent, J.W.; Bruce, D.M. Increased resistance to pod shatter is associated with changes in the vascular structure in pods of a resynthesized Brassica napus line. J. Exp. Bot. 2003, 54, 1919-1930. [CrossRef]

42. Lo Gullo, M.A.; Salleo, S.; Piaceri, E.C.; Rosso, R. Relations between vulnerability to xylem embolism and xylem conduit dimensions in young trees of Quercus corris. Plant Cell Environ. 1995, 18, 661-669. [CrossRef]

43. Trifilo, P.; Lo Gullo, M.A.; Nardini, A.; Pernice, F.; Salleo, S. Rootstock effects on xylem conduit dimensions and vulnerability to cavitation of Olea europaea L. Trees 2007, 21, 549-556. [CrossRef]

44. Meland, M.; Moe, M.E.; Frøynes, O. Differences in growth and development of functional xylem of grafted and budded sweet cherry trees. In Proceedings of the VIII International Symposium on Canopy, Rootstocks and Environmental Physiology in Orchard Systems, Budapest, Hungary, 13-18 June 2004; pp. 311-316.

45. Zach, A.; Schuldt, B.; Brix, S.; Horna, V.; Culmsee, H.; Leuschner, C. Vessel diameter and xylem hydraulic conductivity increase with tree height in tropical rainforest trees in Sulawesi, Indonesia. Flora-Morphol. Distrib. Funct. Ecol. Plants 2010, 205, 506-512. [CrossRef]

46. Tyree, M.T.; Ewers, F.W. The hydraulic architecture of trees and other woody plants. New Phytol. 1991, 119, 345-360. [CrossRef]

47. Şahin-Çevik, M.; Çevik, B.; Topkaya-Kütük, B.; Yazıcı, K. Identification of drought-induced genes from the leaves of Rangpur lime (Citrus limon (L) Osbeck). J. Hortic. Sci. Biotechnol. 2017, 92, 636-645. [CrossRef]

48. Shafqat, W.; Jaskani, M.J.; Maqbool, R.; Chattha, W.S.; Ali, Z.; Naqvi, S.A.; Haider, M.S.; Khan, I.A.; Vincent, C.I. Heat shock protein and aquaporin expression enhance water conserving behavior of citrus under water deficits and high temperature conditions. Environ. Exp. Bot. 2021, 181, 104270. [CrossRef]

49. Ábrahám, E.; Hourton-Cabassa, C.; Erdei, L.; Szabados, L. Methods for determination of proline in plants. In Plant Stress Tolerance; Humana Press-Springer: Totowa, NJ, USA, 2010; pp. 317-331.

50. Velikova, V.; Yordanov, I.; Edreva, A. Oxidative stress and some antioxidant systems in acid rain-treated bean plants: Protective role of exogenous polyamines. Plant Sci. 2000, 151, 59-66. [CrossRef]

51. Hussain, S.; Khalid, M.F.; Saqib, M.; Ahmad, S.; Zafar, W.; Rao, M.J.; Morillon, R.; Anjum, M.A. Drought tolerance in citrus rootstocks is associated with better antioxidant defense mechanism. Acta Physiol. Plant 2018, 40, 135. [CrossRef]

52. Santana-Vieira, D.D.S.; Freschi, L.; da Hora Almeida, L.A.; de Moraes, D.H.S.; Neves, D.M.; Dos Santos, L.M.; Bertolde, F.Z.; dos Santos Soares Filho, W.; Coelho Filho, M.A.; da Silva Gesteira, A. Survival strategies of citrus rootstocks subjected to drought. Sci. Rep. 2016, 6, 38775. [CrossRef] 
53. Aroca, R. Plant responses to drought stress. In From Morphological to Molecular Features; Springer: Berlin/Heidelberg, Germany, 2012.

54. Zhang, J.; Kirkham, M.B. Water status of drought-resistant and drought-sensitive sorghum treated with ethephon. J. Plant Growth Regul. 1990, 9, 189-194. [CrossRef]

55. Savé, R.; Biel, C.; Domingo, R.; Ruiz-Sánchez, M.C.; Torrecillas, A. Some physiological and morphological characteristics of citrus plants for drought resistance. Plant Sci. 1995, 110, 167-172. [CrossRef]

56. Homayoun, H.; Daliri, M.S.; Mehrabi, P. Effect of drought stress on leaf chlorophyll in corn cultivars (Zea mays). Middle East. J. Sci. Res. 2011, 9, 418-420.

57. Mafakheri, A.; Siosemardeh, A.F.; Bahramnejad, B.; Struik, P.C.; Sohrabi, Y. Effect of drought stress on yield, proline and chlorophyll contents in three chickpea cultivars. Aust. J. Crop. Sci. 2010, 4, 580-585.

58. Nawazish, S.; Hameed, M.; Naurin, S. Leaf anatomical adaptations of Cenchrus ciliaris L. from the Salt Range, Pakistan against drought stress. Pak. J. Bot. 2006, 38, 1723-1730.

59. Molassiotis, A.; Job, D.; Ziogas, V.; Tanou, G. Citrus plants: A model system for unlocking the secrets of NO and ROS-inspired priming against salinity and drought. Front. Plant Sci. 2016, 7, 229. [CrossRef] [PubMed]

60. Hayat, S.; Hayat, Q.; Alyemeni, M.N.; Wani, A.S.; Pichtel, J.; Ahmad, A. Role of proline under changing environments: A review. Plant Signal. Behav. 2012, 7, 1456-1466. [CrossRef]

61. Chartzoulakis, K.; Patakas, A.; Bosabalidis, A.M. Changes in water relations, photosynthesis and leaf anatomy induced by intermittent drought in two olive cultivars. Environ. Exp. Bot. 1999, 42, 113-120. [CrossRef]

62. Xiao, J.; Chen, J.; Zhang, H.; Xu, H.; Wang, H.; Xie, M. Gene expression profiling in response to drought stress in citrus leaves by cDNA-AFLP. Acta Hortic. Sin. 2011, 38, 417-424.

63. Wu, Q.-S.; Srivastava, A.K.; Zou, Y.-N. AMF-induced tolerance to drought stress in citrus: A review. Sci. Hortic. 2013, 164, 77-87. [CrossRef]

64. Qaderi, M.M.; Martel, A.B.; Dixon, S.L. Environmental factors influence plant vascular system and water regulation. Plants 2019, 8, 65. [CrossRef] [PubMed]

65. Balfagón, D.; Terán, F.; de Oliveira, T.; Santa-Catarina, C.; Gómez-Cadenas, A. Citrus rootstocks modify scion antioxidant system under drought and heat stress combination. Plant Cell Rep. 2021, 1-10. [CrossRef]

66. Mansoor, U.; Fatima, S.; Hameed, M.; Naseer, M.; Ahmad, M.S.A.; Ashraf, M.; Ahmad, F.; Waseem, M. Structural modifications for drought tolerance in stem and leaves of Cenchrus ciliaris L. ecotypes from the Cholistan Desert. Flora 2019, 261, 151485. [CrossRef]

67. Crous, C.J.; Greyling, I.; Wingfield, M.J. Dissimilar stem and leaf hydraulic traits suggest varying drought tolerance among co-occurring Eucalyptus grandis $\times$ E. urophylla clones. South. For. J. For. Sci. 2018, 80, 175-184. [CrossRef] 